\title{
SUBMICRON-SCALE SURFACE ARCHITECTURE OF TRICALCIUM PHOSPHATE DIRECTS OSTEOGENESIS IN VITRO AND IN VIVO
}

\author{
N.L. Davison ${ }^{1,2, *}$, X. Luo ${ }^{1,2}$, T. Schoenmaker ${ }^{3}$, V. Everts ${ }^{3}$, H. Yuan ${ }^{1,2,4}$, F. Barrère-de Groot ${ }^{2}$ and J.D. de Bruijn ${ }^{12,5}$ \\ ${ }^{1}$ MIRA Institute for Biomedical Technology and Technical Medicine, University of Twente, 7522 NB Enschede, \\ The Netherlands \\ ${ }^{2}$ Xpand Biotechnology BV, 3723 MB Bilthoven, The Netherlands \\ ${ }^{3}$ Departments of Oral Cell Biology and Periodontology, Academic Centre for Dentistry Amsterdam (ACTA), \\ University of Amsterdam and VU University Amsterdam, Research Institute MOVE, 1081 BT Amsterdam, \\ The Netherlands \\ ${ }^{4}$ College of Physical Science and Technology, Sichuan University, Chengdu, Sichuan, P.R. China \\ ${ }^{5}$ School of Engineering and Materials Science (SEMS), Queen Mary University of London, E1 4NS London, \\ United Kingdom
}

\begin{abstract}
A current challenge of synthetic bone graft substitute design is to induce bone formation at a similar rate to its biological resorption, matching bone's intrinsic osteoinductivity and capacity for remodelling. We hypothesise that both osteoinduction and resorption can be achieved by altering surface microstructure of beta-tricalcium phosphate (TCP). To test this, two TCP ceramics are engineered with equivalent chemistry and macrostructure but with either submicron- or micron-scale surface architecture. In vitro, submicron-scale surface architecture differentiates larger, more active osteoclasts - a cell type shown to be important for both TCP resorption and osteogenesis - and enhances their secretion of osteogenic factors to induce osteoblast differentiation of human mesenchymal stem cells. In an intramuscular model, submicrostructured TCP forms $20 \%$ bone in the free space, is resorbed by $24 \%$, and is densely populated by multinucleated osteoclast-like cells after 12 weeks; however, TCP with micron-scale surface architecture forms no bone, is essentially not resorbed, and contains scarce osteoclast-like cells. Thus, a novel submicron-structured TCP induces substantial bone formation and is resorbed at an equivalent rate, potentially through the control of osteoclast-like cells.
\end{abstract}

Keywords: Calcium phosphate, topography, microstructure, osteoclast, osteogenesis.

*Address for correspondence:

Noel Davison

Xpand Biotechnology BV

3723 MB Bilthoven, The Netherlands

FAX Number: +31(0)30 2297299

E-mail: Noel.Davison@Xpand-Biotech.com

\section{Introduction}

A novel approach to regenerative biomaterial design is to engineer instructive physiologic signals to stimulate tissue repair. In the case of synthetic materials used for repairing bone tissue, some of the most widely used clinically are calcium phosphates $(\mathrm{CaP})$, which are well tolerated by the body due to their chemical homology to native bone mineral (Bohner et al., 2012). CaP provide a particularly flexible platform for material design because the physicochemical and topographical characteristics can be tuned by modifying their synthesis parameters (Osborn and Newesely, 1980; Famery et al., 1994; Dorozhkin, 2010). Of these materials, a small subset can stimulate the formation of bone even in heterotopic, non-bony locations without exogenous cells or growth factors (LeGeros, 2008; Barradas et al., 2011). Such osteoinductive CaP have been shown to be superior to non-inductive (e.g., merely osteoconductive) materials for repairing critical size bony defects that exceed the natural repair capacity of the bone organ (Habibovic et al., 2006; Ripamonti et al., 2008; Yuan et al., 2010; Akiyama et al., 2011).

The purposeful design of an osteoinductive $\mathrm{CaP}$ is an elusive challenge for materials science because the material parameters necessary to instruct osteogenesis are still critically undefined. In an iterative approach spanning more than 20 years, macrostructural elements such as three-dimensional architecture (Yuan et al., 2001b; Habibovic et al., 2005), surface concavities (Ripamonti et al., 2008), and interconnected pore structure (Yuan et al., 1999; Fukuda et al., 2011) were all shown to enhance bone formation by CaP. Other materials such as Bioglass (Yuan et al., 2001b) and titanium (Fujibayashi et al., 2004) have also been shown in some scattered studies to induce small amounts of ectopic bone formation. Most recently, the surface microstructure has been suggested to have a directive effect on ectopic bone formation in intramuscular models (Fujibayashi et al., 2004; Le Nihouannen et al., 2005; Habibovic et al., 2006; Ripamonti et al., 2008; Yuan et al., 2010). For instance, bioactivity may occur at the material surface where ions and proteins are absorbed from the blood. It is speculated that augmenting the specific surface area by changing the surface architecture, e.g., increasing micropore volume, may increase reprecipitation/release of $\mathrm{Ca}^{2+}$ and absorption of bone morphogenetic proteins (BMPs), both of which 
can stimulate the osteogenic differentiation of stem cells (Barradas et al., 2011).

Beyond physico-chemical effects, osteoinductive surface architecture may also confer powerful cell-fate regulating signals directly to adherent cells through cell-substrate interactions via mechanotransduction. For example, osteoblast genes in human mesenchymal stem cells (hMSC) are upregulated following culture on osteoinductive CaP surfaces in osteogenic conditions (Yuan et al., 2010), presumably due to the topographical cues transduced by cell adhesion molecules. Similar effects have been described on polymer substrates with submicronand nano-scale surface features even without osteogenic additives, suggesting that instructive surface architecture can induce osteogenesis irrespective of surface chemistry when it is engineered on a certain size scale (Dalby et al., 2006; Dalby et al., 2007).

Another important aspect of material design for bone regeneration is the resorbability of the implant. Because bone itself is a continuously remodelled material, resulting from the tightly coupled interplay between specialised bone-resorbing osteoclasts and bone-secreting osteoblasts (Sims and Gooi, 2008), a synthetic bone substitute that can also be physiologically remodelled or resorbed would provide a more biomimetic scaffold for bony ingrowth, deposition, and long term homeostasis. If a material is non-resorbable, there is a risk that the mechanical loading will be distributed unequally due to stress shielding of the implant, weakening the less loaded surrounding bone over time. Consequently, because $\beta$-tricalcium phosphates (TCP) have been shown to be highly resorbable both clinically and in vitro, they are preferred versus hydroxyapatites, which are mostly non-resorbable (Blokhuis et al., 2000). The proposed mechanisms of $\mathrm{CaP}$ resorption are diverse and include passive dissolution, hydrolysis, and cell-mediated dissolution. For instance, it is speculated that osteoclasts play a principal role in the cellular resorption and this can be evaluated by characteristic osteoclastic resorption pits in vitro (Yamada et al., 1997; Monchau et al., 2002). Because of the multifactorial nature of the physiologic phenomenon, the only true test to evaluate material resorbability is to evaluate the bulk loss of material in vivo (Bohner et al., 2012). Still, understanding the dominant mechanism of resorption would prove useful for the design of better TCP.

Though the cellular events leading to de novo bone formation by $\mathrm{CaP}$ are largely unknown, it has been demonstrated in several studies that osteoclasts may play an important role. For instance the formation of osteoclasts has been found to precede de novo bone formation by osteoinductive TCP in the dorsal muscle of dogs by four weeks (Kondo et al., 2006). Moreover, studies by Ripamonti et al. (2010) and Tanaka et al. (2010) published in the same year showed that administering osteoclastinhibiting bisphosphonate reduced the bone formation of two different osteoinductive ceramics (hydroxyapatite/ calcium carbonate and TCP) in both heterotopic and orthotopic sites. One potential explanation for these findings is that osteoclasts express and secrete osteogenic factors such as BMPs (McCullough et al., 2007; Pederson et al., 2008) and that surface architecture - in particular surface roughness - can profoundly influence their development and function (Marchisio et al., 2005; CostaRodrigues et al., 2012).

Although CaP provide a flexible platform for designing material chemistry and architecture, the fine control of one variable at a time while maintaining the other properties static continues to be a challenge for materials science. Indeed, altering surface architecture, i.e. surface micropore and grain size, consequently changes other surface properties that stem from it, including specific surface area, surface reactivity and physical topography; as such, these factors are inextricably linked. Nevertheless, whether there is an instructive size scale of surface architecture necessary to trigger both bone induction coupled with an equivalent amount of material resorption remains unknown. The aim of this work was thus to tune the surface microstructure while keeping the material chemistry and macrostructure constant with the end goal of designing a resorbable $\mathrm{CaP}$ with instructive surface features to stimulate osteogenesis. We hypothesised that the scale of surface features plays a determinant role in this outcome because cellular behaviour has been shown to be powerfully influenced at the material surface interface (Stevens and George, 2005). To test this, two TCP with either micron-scale or submicronscale surface features and equivalent chemistries were carefully synthesised and their physico-chemical properties were characterised. The effect of different scale surface architecture on osteoclast formation and activity - in particular secreted osteogenic signals - was evaluated in vitro and the bone inductive capacity and resorbability of the materials was evaluated in vivo.

\section{Materials and Methods}

\section{Preparation and characterisation of TCP with} micron- and submicron-scale surface architecture TCP powders were synthesised by mixing calcium hydroxide and phosphoric acid (both from Fluka/SigmaAldrich, St. Louis, MO, USA) at a $\mathrm{Ca} / \mathrm{P}$ ratio of 1.50 . TCP powders with small (TCPs) or big grains $(\mathrm{TCPb})$ in the final ceramics were prepared by controlling the reaction rates. The powders were foamed with diluted $\mathrm{H}_{2} \mathrm{O}_{2}(1 \%)$ (Merck, Darmstadt, Germany) at $60{ }^{\circ} \mathrm{C}$ to get macro/microporous green bodies; meanwhile the powders were mixed with diluted $\mathrm{H}_{2} \mathrm{O}_{2}(0.1 \%)$ and kept dried at room temperature to get microporous green bodies. The dry green bodies (both macro/micro and microporous ones) were subsequently sintered at $1050{ }^{\circ} \mathrm{C}$ or $1100{ }^{\circ} \mathrm{C}$ for $8 \mathrm{~h}$ to achieve small and big grains for TCPs and $\mathrm{TCPb}$, all respectively.

Macro/microporous cylinders $(\varnothing 7 \times 10 \mathrm{~mm})$ and microporous discs $(\varnothing 9 \times 1 \mathrm{~mm})$ were machined from the ceramic bodies using a lathe and a diamond-coated saw microtome (Leica SP1600; Leica, Solms, Germany) and ultrasonically cleaned, and heat sterilised at $160{ }^{\circ} \mathrm{C}$ for $2 \mathrm{~h}$. Macro/microporous cylinders were used for animal implantation to allow for quantification of bone formation and implant resorption, whereas microporous discs were used for in vitro experiments to avoid effects of three-dimensional macrostructure, i.e., variability of cell seeding, cell infiltration into the material, incomplete cell lysis, and limitations visualising cells by microscopy. 
Crystal chemistry of the materials was analysed by X-ray diffraction (Rigaku Miniflex II; Rigaku, Seven Oaks (Kent), UK) scanning the range $2 \theta=25-45^{\circ}$ (step size $=0.01^{\circ}$, rate $=1^{\circ} \mathrm{min}^{-1}$ ) and confirmed to be TCP as previously described (Davison et al., 2012).

Surface topography of the materials was characterised using scanning electron microscopy (SEM) (JEOL JSM5600; JEOL, Tokyo, Japan) after sputter coating them with gold for $90 \mathrm{~s}$ (JEOL JFC 1300). At least 50 grains and pores were measured across their vertical diameter using Image J software (NIH, Bethesda, MD, USA). Porosity and total pore area were determined by mercury intrusion testing (Table 1) (Micromeritics, Atlanta, GA, USA). For surface profile characterisation including calculation of surface roughness parameters, SEM stereo-micrographs of the same location were captured at two different tilt angles $\left(2500 \mathrm{x}, \pm 5^{\circ}\right)$ and then digitally reconstructed into three-dimensional surfaces for automated profile analysis using MeX v5.1 software (Alicona Imaging, www.alicona. com).

Surface reactivity of the materials was evaluated during incubation in complete cell culture medium (described below) at $37{ }^{\circ} \mathrm{C}$ and $5 \% \mathrm{CO}_{2}$ for up to 14 days to mimic a physiologic environment. Medium (1 mL) incubated with TCP discs was collected and refreshed at several time points $(3,7,10$ and $14 \mathrm{~d})$ and assayed for $\mathrm{Ca}^{2+}$ and inorganic phosphate $\left(\mathrm{P}_{\mathrm{i}}\right)$ concentrations using QuantiChrom (BioAssay Systems, Hayward, CA, USA) and PhoshoWorks (AAT BioQuest, Sunnyvale, CA, USA) colorimetric biochemical kits, respectively. Medium incubated without TCP served as control to calculate the mass of ions absorbed by the materials. At each time point, ion concentrations measured in medium incubated with TCP were subtracted from those measured in the control medium, yielding the ion mass absorbed by TCP. Cumulative absorption was computed by summing the ion absorption at all previous time points in a compounding fashion. The media from $n=3$ TCP disc replicates was measured at each time point.

\section{In vivo study}

Intramuscular implantation

All surgery was conducted at the Animal Centre of Sichuan University in conformance with the institutional animal ethics committee's guidelines. Sterile TCP macro/ microporous cylinders were implanted in the dorsal muscle of healthy male mongrel dogs $(n=8,1-4$ years, $10-15 \mathrm{~kg}$ ) for 12 weeks. Animals were first given general anaesthesia by abdominal injection of sodium pentobarbital (30 mg kg-1 body weight) and cylinders were implanted into paraspinal muscle pockets created by scalpel incision and blunt dissection. Skin incisions were closed layer by layer with non-resorbable sutures for identification at harvest. Following surgery, the animals were given daily intramuscular injections of buprenorphine $(0.1 \mathrm{mg}$ per animal) for $2 \mathrm{~d}$ and penicillin $\left(40 \mathrm{mg} \mathrm{kg}^{-1}\right)$ for $3 \mathrm{~d}$ to relieve pain and prevent infection. Animals were allowed to undertake full activity and received a normal diet immediately after surgery.

\section{Sample harvest and histological processing}

At the end of 12 weeks the animals were sacrificed by abdominal injection of sodium pentobarbital $\left(60 \mathrm{mg} \mathrm{kg}^{-1}\right)$ and samples were immediately harvested and fixed in cold phosphate-buffered formalin solution, dehydrated in graded ethanol series, and embedded in methyl methacrylate (MMA) (LTI, Dutch Polymer Institute, The Netherlands, www.polymers.nl) at room temperature.

Histological sections (approx. $20 \mu \mathrm{m}$ ) of the undecalcified samples were made using a Leica SP1600 microtome, stained en bloc with $1 \%$ methylene blue and $0.3 \%$ basic fuchsin solutions for histological analysis and histomorphometry.

\section{Histology and histomorphometry}

Stained histological sections were scanned using a Dimage Scan Elite 5400II slide scanner (Konica Minolta, Tokyo, Japan) for gross evaluation and histomorphometric analysis, as well as at higher magnifications using a light

Table 1. Surface characterisation and roughness profile of TCP

\begin{tabular}{|l|c|c|}
\hline & TCPs & TCPb \\
\hline Average grain diameter $(\boldsymbol{\mu m})$ & $0.95 \pm 0.27$ & $3.66 \pm 1.05$ \\
\hline Average pore diameter $(\boldsymbol{\mu m})$ & $0.63 \pm 0.33$ & $1.78 \pm 0.85$ \\
\hline Average peak-to-valley roughness, $\mathbf{R}_{\mathbf{a}}(\boldsymbol{\mu m})$ & $0.126 \pm 0.003$ & $1.287 \pm 0.011$ \\
\hline Root-mean-square peak-to-valley roughness, $\mathbf{R}_{\mathbf{R M S}}(\boldsymbol{\mu m})$ & $0.158 \pm 0.003$ & $1.597 \pm 0.011$ \\
\hline
\end{tabular}

Table 2. Porosity by mercury intrusion testing

\begin{tabular}{|c|c|c|c|c|}
\hline & \multicolumn{2}{|c|}{ Microporous Discs } & \multicolumn{2}{c|}{ Macro/Microporous Cylinders } \\
\hline & TCPs & TCPb & TCPs & TCPb \\
\hline Total pore area $\left(\mathbf{m}^{2} / \mathbf{g}\right)$ & 1.424 & 0.521 & 1.477 & 0.769 \\
\hline Total Porosity $(\%)$ & 46.3 & 49.8 & 69.6 & 72.0 \\
\hline
\end{tabular}


microscope (Nikon Eclipse E200; Nikon, Tokyo, Japan).

Histomorphometry of bone formation and TCP resorption in the stained sections was performed by pseudo-colouring pixels representing formed bone (B) and remaining material $(\mathrm{M})$ in a region of interest (ROI) using photo analysis software (Adobe Photoshop Elements 4.0; Adobe, San José, CA, USA). The percent area of bone formed $(B \%)$ in the available space was then calculated by the equation:

$$
B \%=\mathrm{B} /(\mathrm{ROI}-\mathrm{M}) \times 100
$$

The percent area of material resorbed ( $\mathrm{M} \%$ ) was calculated by subtracting the pixel area of remaining material, $\mathrm{M}$, from the mean pixel area of similarly embedded, sectioned, and pseudo-collared TCP cylinders $(n=3)$ that had not been implanted $\left(\mathrm{M}_{\mathrm{o}}\right)$ :

$$
M \%=\left(\mathrm{M}_{\mathrm{o}}-\mathrm{M}\right) / \mathrm{M}_{0} \times 100
$$

\section{In vitro studies}

RAW264.7 monocyte/macrophage culture on TCP discs Murine monocyte/macrophage cell line RAW264.7 (ECACC, Wiltshire, UK) was expanded in basic medium composed of alpha MEM (Lonza, Basel, Switzerland), supplemented with $10 \%$ HyClone FetalClone I serum (Thermo Scientific) and $1 \%$ penicillin-streptomycin (Life Technologies, Carlsbad, CA, USA). Near-confluent cells were detached by scraping and seeded on TCP discs $\left(2 \times 10^{4}\right.$ cells $\left.\mathrm{cm}^{-2}\right)$ in non-tissue culture treated 48-well plates containing basic medium supplemented with RANKL (40 ng mL ${ }^{-1}$, Peprotech, Rocky Hill, NJ, USA) to induce osteoclast formation. TCP discs incubated in basic medium +/- RANKL without cells served as acellular controls. After $5 \mathrm{~d}$ of culture, conditioned medium was collected from $n=5$ replicate discs, centrifuged to remove cellular debris, and frozen.

\section{Human mesenchymal stem cell culture in conditioned media}

Human mesenchymal stem cells (hMSC) were isolated from bone marrow biopsies obtained from the femoral head of 3 patients (68-80 years) undergoing total hip replacement surgery (Albert Schweitzer Hospital, Dordrecht, The Netherlands, approved under Medical Ethical Committee trial number 2011.07) by density gradient isolation of the buffy coat. Adherent cells were expanded in flasks containing growth medium: basic medium supplemented with L-glutamine (2 mM) (GlutaMAX I, Fisher Scientific, Waltham, MA, USA), L-ascorbic acid 2-phosphate (0.2 mM) (Sigma-Aldrich), and basic fibroblast growth factor $\left(1 \mathrm{ng} \mathrm{mL}^{-1}\right)$ (Serotech, Oxford, UK); non-adherent cells were removed in subsequent media refreshments. Twice-passaged hMSC were trypsinised and seeded in 96well plates containing basic medium $\left(1 \times 10^{4}\right.$ cells $\left.\mathrm{cm}^{-2}\right)$. After one day, hMSC were supplemented with conditioned medium $(50 \mu \mathrm{L})$ and refreshed with the same amount every other day for $7 \mathrm{~d}$ total. All culture was maintained at $37{ }^{\circ} \mathrm{C}, 100 \%$ humidity, and $5 \% \mathrm{CO}_{2} . n=4$ replicates were cultured per condition, per hMSC donor.

\section{TRAP staining}

The presence of the osteoclast enzyme tartrate resistant acid phosphatase (TRAP) was detected using a commercial staining kit (Leukocyte Acid Phosphatase Kit, SigmaAldrich). Staining was visualised using a Nikon SMZ800 stereomicroscope equipped with a Nikon camera. $n=2$ replicate discs were stained and analysed per condition.

\section{Biochemical activity}

For biochemical assays on the cell lysate, cells were lysed in a commercial lysis buffer (Promega, Madison, WI, USA). TRAP activity was quantified in the cell lysate of RANKL-stimulated RAW264.7 by conversion of $p$-nitrophenylphosphate to $p$-nitrophenol (NP) in sodium acetate buffer ( $\mathrm{pH} 5.8$ ) containing potassium sodium tartrate $(10 \mathrm{mM})$ as reported by Ljusberg et al. (1999). Alkaline phosphatase (ALP) activity was measured in the hMSC lysate using the AttoPhos Alkaline Phosphatase Fluorescent Substrate kit (Promega, Madison, WI, USA). Relative ALP was normalised to the basal ALP level measured in the negative control: hMSC cultured in basic medium; hMSC cultured with osteogenic supplements dexamethasone $(10 \mathrm{nM})$ and L-ascorbic 2-phosphate $(0.2 \mathrm{mM})$ served as the positive control reference. Both TRAP and ALP were normalised to DNA content in the same lysate using a CyQuant DNA detection kit (Life Technologies). A Zenyth 3100 Multimode plate reader (Biochrom, Cambridge, UK) was used to detect optical density and fluorescent signal of the assays. $n=4$ replicate discs were analysed per condition.

Fluorescent confocal microscopy of osteoclast formation Osteoclast fusion and actin ring formation were analysed using fluorescent confocal microscopy. Osteoclast-cultured discs were rinsed with PBS, fixed in $4 \%$ formaldehyde for $48 \mathrm{~h}$, blocked with $20 \%$ normal goat serum, then sequentially incubated with rat anti-mouse monoclonal primary antibody CD44 targeting the osteoclast plasma membrane (1:100 dilution) (Cedarlane, Burlington, Ont., Canada), goat anti-rat Alexa467-conjugated secondary antibody (1:400) (Invitrogen/Life Technologies), Alexa488conjugated phalloidin targeting F-actin (1:400) (Invitrogen/ Life Technologies), and propidium iodide (1:1000) (SigmaAldrich) to stain nuclei, as previously described (Jansen et al., 2012). Fluorescence was visualised using a Leica TCS-SP2 laser scanning confocal microscope (Leica) and image stacks were generated by scanning from the apical to basal surface of the cell and overlaid. $n=2$ replicate discs were stained and analysed.

\section{SEM of osteoclast morphology}

Osteoclast morphology was evaluated using SEM (JEOL JSM-5600). RANKL-treated RAW264.7 were fixed in $2.5 \%$ glutaraldehyde, dehydrated in a graded ethanol series and finally dried in HMDS (Alfa Aesar; www.alfa. com). Dehydrated cells were then sputter coated with gold for enhanced imaging resolution. Osteoclast size was quantified in SEM micrographs (x 100) by calculating the mean surface area of cells at 3 random locations per disc using automated threshold, edge detection, and particle 
analysis functions in Image J software, as previously described (Dalby et al., 2004). Only cells whose area was $>200 \mu \mathrm{m}^{2}$ were included in the analysis to safely exclude mononuclear cells (Theiss et al., 2005). $n=3$ replicate discs were analysed per condition.

\section{Osteoclast gene expression by quantitative PCR}

Total RNA was isolated for qPCR analysis using TRIzol reagent (Life Technologies) according to the manufacturer's instructions. RNA from $n=3$ replicate discs was isolated and pooled each day through 5 days of culture. The RNA concentration was measured using a Synergy spectrophotometer (Biotek, Winooski, VT, USA). Reverse transcription of $6 \mu \mathrm{g}$ RNA was performed using the MBI Fermentas cDNA synthesis kit (Fermentas/ Thermo Scientific) and accompanying Oligo(dT)18 and $\mathrm{D}(\mathrm{N}) 6$ primers.

Quantitative PCR (qPCR) was performed on an ABI (Applied Biosystems/Life Technologies) PRISM 7000 Sequence Detection System. The PCR reactions were performed with $60 \mathrm{ng}$ cDNA in a total volume of $15 \mu \mathrm{L}$ containing SYBR GreenER qPCR SuperMix, consisting of SYBR Green 1 Dye, AmpliTaq Gold DNA polymerase, dNTPs, passive reference and buffer (Life Technologies) and $300 \mathrm{~nm}$ of each primer. After an initial activation step for $10 \mathrm{~min}$ at $94{ }^{\circ} \mathrm{C}, 40$ cycles were run of a twostep PCR consisting of a denaturation step at $95{ }^{\circ} \mathrm{C}$ for $15 \mathrm{~s}$ and annealing and extension step at $60{ }^{\circ} \mathrm{C}$ for $1 \mathrm{~min}$. Subsequently, the PCR products were subjected to melting curve analysis to test if any unspecific PCR products were generated.

QPCR primers were designed using Primer Express 2.0 software (Life Technologies), spanning at least 1 intron to avoid amplification of genomic DNA. Expression of housekeeping genes $\beta 2$ microglobulin and porphobilinogen deaminase (PBGD) was not affected by the experimental conditions. Expression levels of gene targets were normalised to endogenous $\beta 2$ microglobulin, and then normalised to the negative control (unstimulated RAW264.7 cultured on tissue culture plastic) at each time point (i.e., the $\Delta \Delta \mathrm{Ct}$ method), resulting in relative fold expression.

\section{Statistics}

Statistical comparisons were performed using One-way analysis of variance (ANOVA) and Tukey's post hoc tests; $p$ values $<0.05$ were considered significant. Grain and pore size frequency distributions were fit by nonlinear regression analysis using $\log$ (Gaussian) model for non-normal distributions. All statistical analyses were conducted in Graphpad (LaJolla, CA, USA) Prism 6.0.

\section{Results}

\section{Characterisation of micro- and submicro-structured TCP}

Analysis of SEM micrographs (Fig. 1a,b) and 3D-reconstructed stereo-micrographs (Fig. 1c,d) clearly confirmed the synthesis of two micro-structurally different TCP (Fig. 1a,b). The surface profile of TCPs followed a quasi-regular sinusoidal pattern, with peaks and valleys spanning a submicron scale range, versus that of $\mathrm{TCPb}$ which spanned a micron-scale range (Fig. 1g). These differences were quantified in terms of average $\left(R_{a}\right)$ and root-mean-square $\left(R_{R M S}\right)$ peak to valley height roughness parameters: $126 \pm 3 \mathrm{~nm}$ and $158 \pm 3 \mathrm{~nm}$, respectively for TCPs, versus $1287 \pm 11 \mathrm{~nm}$ and $1597 \pm 11 \mathrm{~nm}$, respectively for $\mathrm{TCPb}$ (Table 1). Quantification of the grains and pores in SEM micrographs showed that both features were on average $<1 \mu \mathrm{m}$ for TCPs and $>1$ peak to valley height roughness parameters: $126 \pm 3 \mathrm{~nm}$ and $158 \pm 3 \mathrm{~nm}$, respectively for TCPs, versus $1287 \pm 11 \mathrm{~nm}$ and $1597 \pm 11 \mathrm{~mm}$, respectively for $\mathrm{TCPb}$ (Table 1). Quantification of the grains and pores in SEM micrographs resulted in submicron-scale surface structure and $\mathrm{TCPb}$ resulted in micron-scale surface structure (Fig. 1e,f).

Though total porosity of TCPs and TCPb was similar ( $\sim 46-50 \%$ for microporous discs; $\sim 69-72 \%$ for macro/ microporous cylinders), total pore area was roughly 2 times greater for TCPs than $\mathrm{TCPb}$, owing to smaller pores but equal overall porosity (Table 2).

To test the surface reactivity in physiologic conditions, the materials were incubated for up to $14 \mathrm{~d}$ in cell culture medium containing serum to simulate blood plasma. $\mathrm{P}_{\mathrm{i}}$ and $\mathrm{Ca}^{2+}$ concentrations in the medium decreased over time, indicating that these ions were continuously absorbed by the materials and not released; however, ion absorption of the materials was substantially different throughout the incubation period presumably due to differences in specific surface area, a consequence of different surface architecture (Fig. 1h,i).

\section{Effects of submicron-scale TCP surface architecture on osteoclast formation and activity in vitro}

Submicron-scale surface structure promotes larger, more multinucleated osteoclasts

It was speculated that differences in surface microstructure modulate the formation of osteoclasts pertinent to osteogenesis by TCP. To test this, the murine monocyte/ macrophage RAW264.7 cells were cultured on TCP discs in the presence of RANKL (Receptor Activator of NF- $\kappa \mathrm{B}$ Ligand) to stimulate the differentiation of osteoclasts. Because these discs lacked macroporosity, surface micropore size restricted cells only to surface interactions and not material infiltration. SEM micrographs confirmed that mononuclear macrophages fused to form multinucleated osteoclast-like cells on the surface of both materials by day 3 . However, at this time, osteoclast fusion on $\mathrm{TCPb}$ appeared to be less complete than on TCPs across disc replicates, illustrated by fragile gaps in the cell membranes between fusing cells (Fig. 2) not observed on TCPs despite identical sample preparation. After $4 \mathrm{~d}$ in culture, distinct differences could be seen in terms of osteoclast size, morphology, and integration with the material surface (Fig. 3). Prominent filopodia stretched out between osteoclasts, connecting them in a highly networked cell population on TCPs (Fig. 3a,b). At higher magnifications, cell membranes were tightly integrated with the material, and the apical sides were decorated by microvilli (Fig. 3c). Conversely, osteoclasts formed on $\mathrm{TCPb}$ (Fig. 3d,e) were far less connected by less frequent 

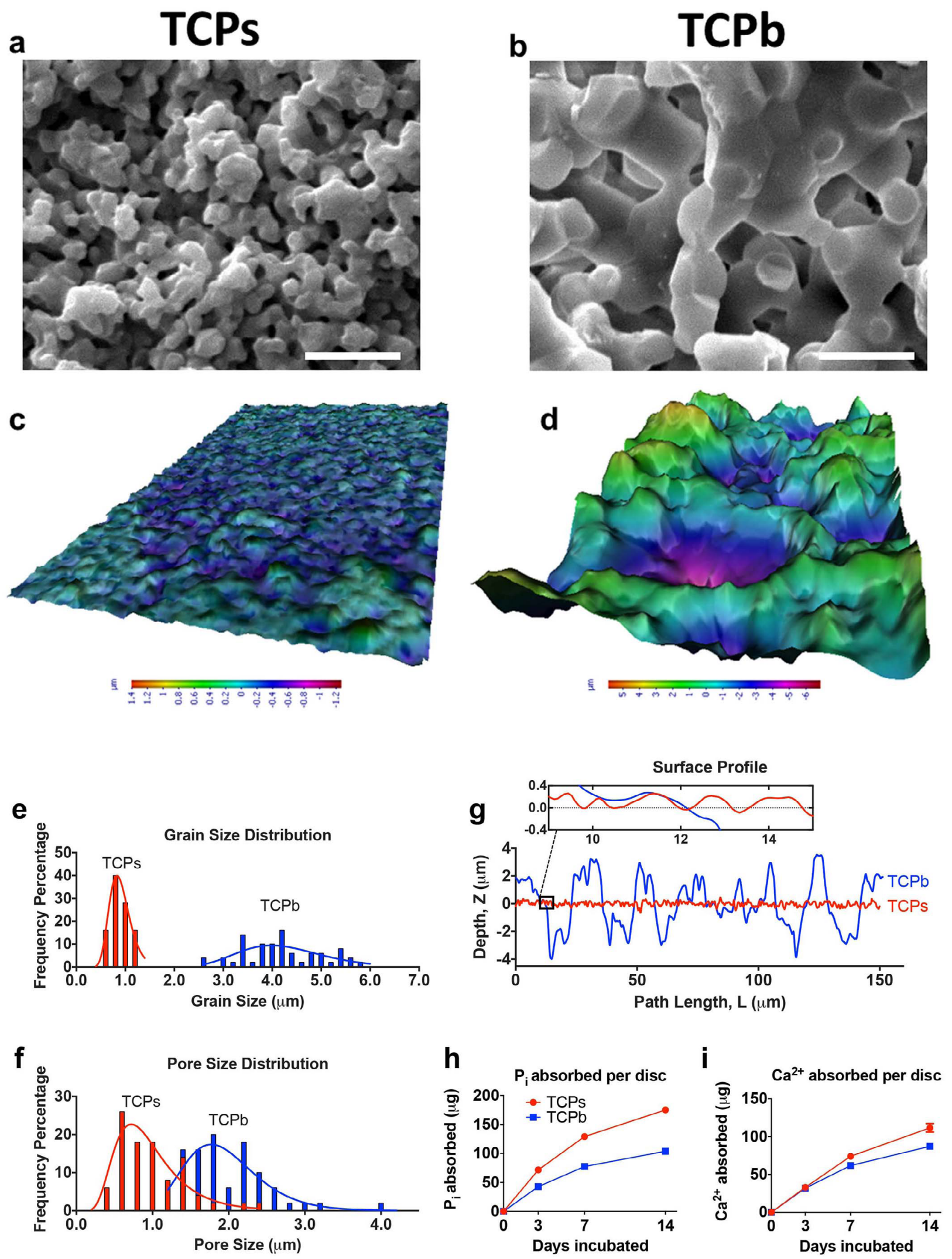

Fig. 1. Characterisation of TCP physico-chemistry. (a, b) SEM micrographs depict different surface microstructure of microporous TCPs and $\mathrm{TCPb}$ (scale bars $=5 \mu \mathrm{m}$ ). (c, d) 3D surface reconstruction, (g) corresponding surface profiles, $(\mathbf{e}, \mathbf{f})$ and grain/pore size distribution emphasise the submicron-scale versus micron-scale architecture of TCPs and TCPb, respectively. Surface reactivity of TCPs and TCPb discs as measured by cumulative (h) $\mathrm{P}_{i}$ and (i) $\mathrm{Ca}^{2+}$ absorption during incubation in serum-containing medium (mean \pm s.d. of $n=3$ discs). 


\section{TCPs}
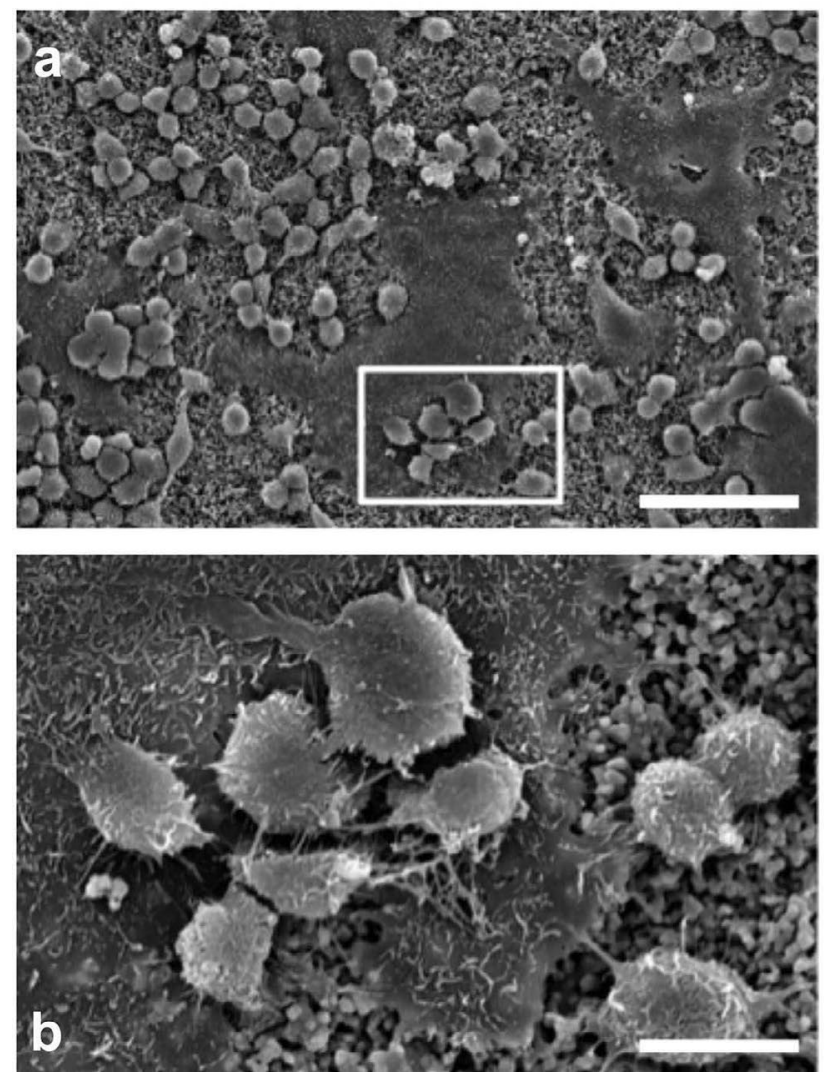

$\mathrm{TCPb}$
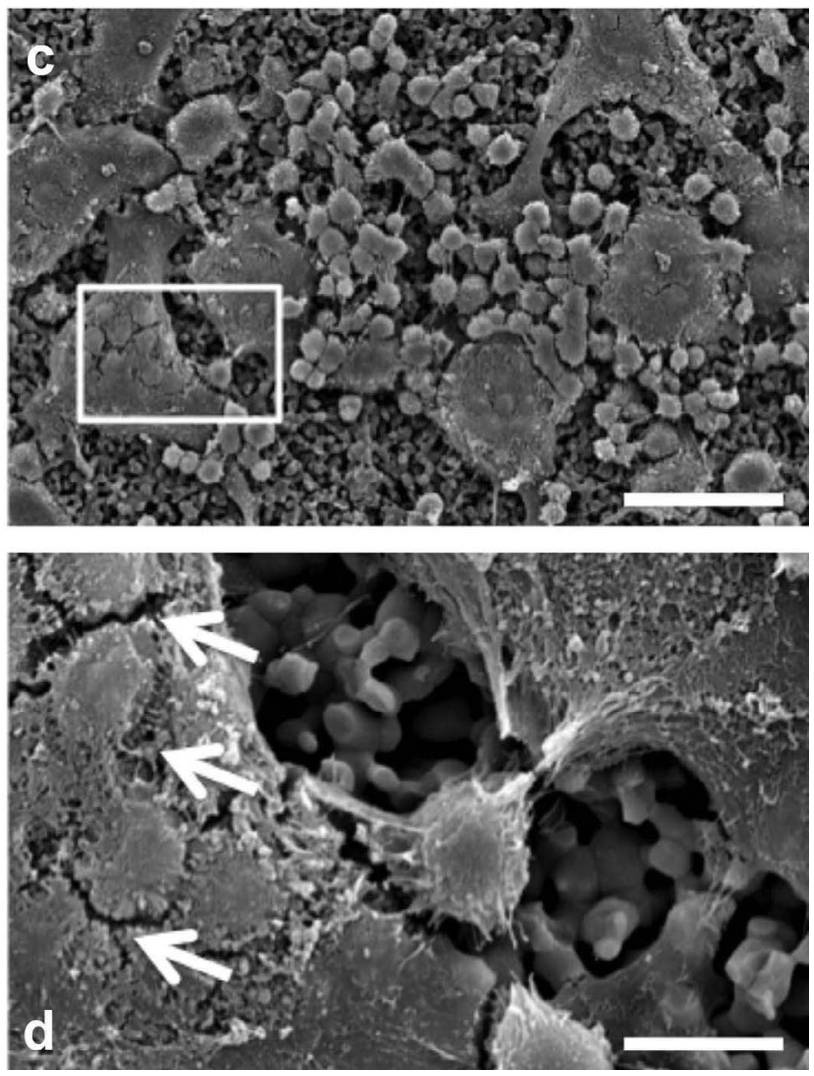

Fig. 2. SEM micrographs of osteoclast formation on $(\mathbf{a}, \mathbf{b}) \mathrm{TCPs}$ and $(\mathbf{c}, \mathbf{d}) \mathrm{TCPb}$ after 3 days of culture. Incomplete cell fusion on $\mathrm{TCPb}$ was implicated by fragile cell membrane gaps (arrows), compared to complete membrane closure and active fusion on TCPs. Scale bars: $\mathbf{a}, \mathbf{c}=50 \mu \mathrm{m} ; \mathbf{b}, \mathbf{d}=10 \mu \mathrm{m}$.

filopodia, and appeared rounder and less integrated with the surface at higher magnifications (Fig. 3f).

Osteoclast size was quantified in SEM micrographs captured at day 4 of culture, which was the concurrent peak of osteoclast fusion and TRAP activity, described in detail below. Osteoclasts formed on TCPs were on average more than twice as large those formed on $\mathrm{TCPb}(p=0.023)$ (Fig. $3 \mathrm{~g}$ ) in some cases encompassing $>1,000 \mu^{2}$. By day 5 , osteoclasts were observed to be less actively fusing and appeared in some cases apoptotic (not shown), indicating that 4 days culture was the peak of osteoclast formation in this culture system.

Cell membrane and nuclei staining visualised by fluorescent confocal microscopy confirmed the trends observed in SEM: osteoclasts formed on TCPs were clearly larger, owing to more fusion and multinucleation (Fig. 4). Membrane staining in particular confirmed the presence of massive networks of fused osteoclasts on TCPs (Fig. $4 \mathrm{a})$, in contrast to isolated, smaller osteoclasts formed on $\mathrm{TCPb}$ (Fig. 4c), which were also multinucleated but to a far lesser extent. Actin rings visualised by F-actin staining were of similar size and number on both materials (Fig. $4 \mathrm{~b}, \mathrm{~d})$, suggesting that these cytoskeletal rearrangements indicative of mature osteoclasts proceeded without dependence on the scale of surface structure tested here.
Submicron-scale surface architecture promotes osteoclast TRAP activity

Surface microstructure was also found to affect osteoclast differentiation and activation indicated by TRAP activity (Fig. 5). On submicrostructured TCPs, TRAP per DNA in the cell lysate of disc-adherent osteoclasts peaked after $4 \mathrm{~d}$ in culture, which was $\sim 25 \%$ higher than on $\mathrm{TCPb}$ $(p=0.001)$ - in contrast, TRAP activity did not increase from day 3 through day 5 of culture on $\mathrm{TCPb}$ (Fig. 5a). TRAP staining consistently confirmed these results with intense TRAP positive staining on TCPs versus low TRAP activity on TCPb (Fig. 5.c-f). Considering cell proliferation, DNA content similarly plateaued by day 4 on both materials due to osteoclast differentiation (Fig. 5b). Thus, different surface microstructure affected the TRAP activity but not cell proliferation.

\section{Submicron-scale surface architecture amplifies osteoclast-secreted osteogenic signals}

Conditioned medium from osteoclast cultures on TCP discs with RANKL was administered to hMSC cultured in basic medium, and ALP activity in the hMSC cell lysate was measured as an indicator of osteoblast differentiation. After one week of culture, hMSC ALP activity (Fig. 6) was $50 \%$ higher than in hMSC treated with conditioned 

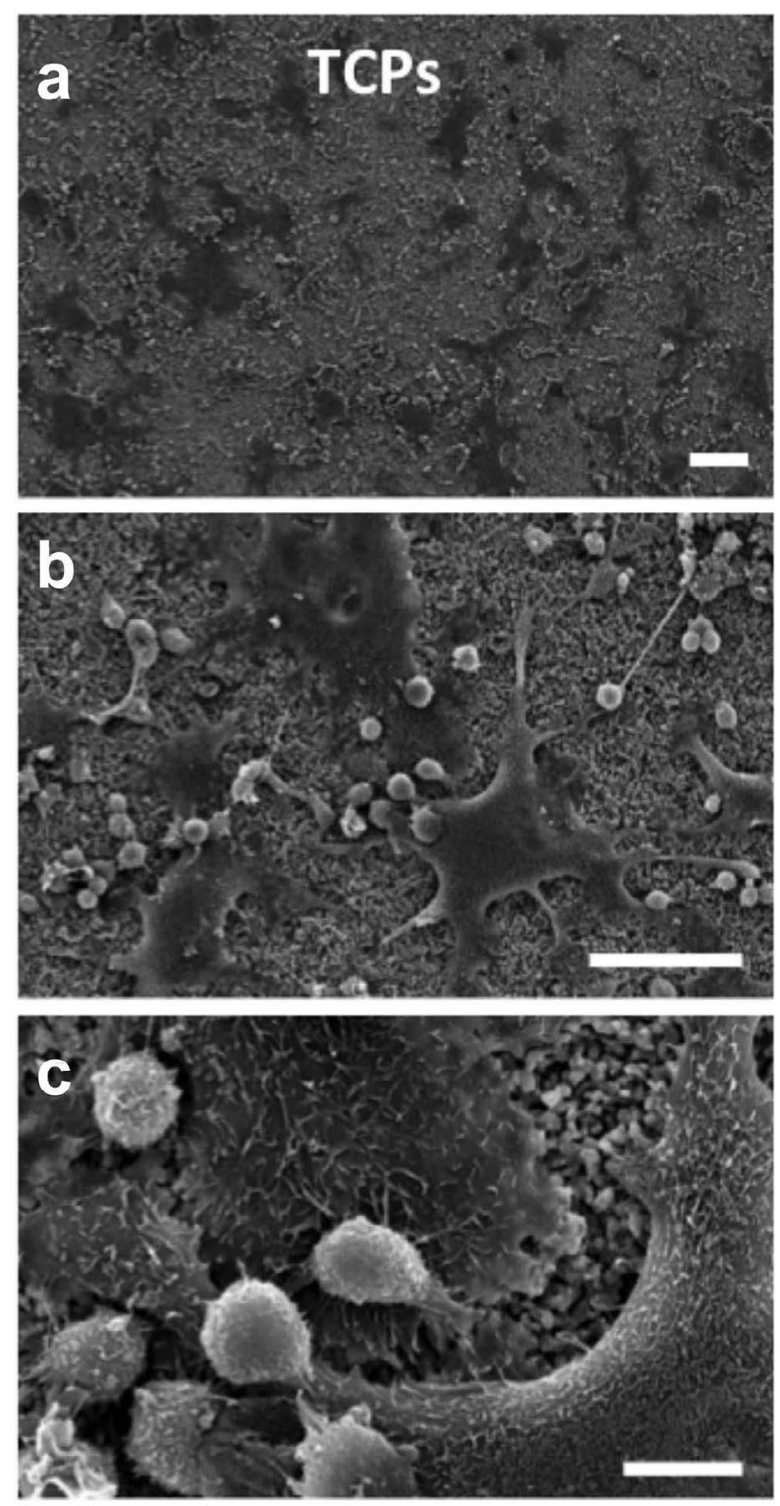
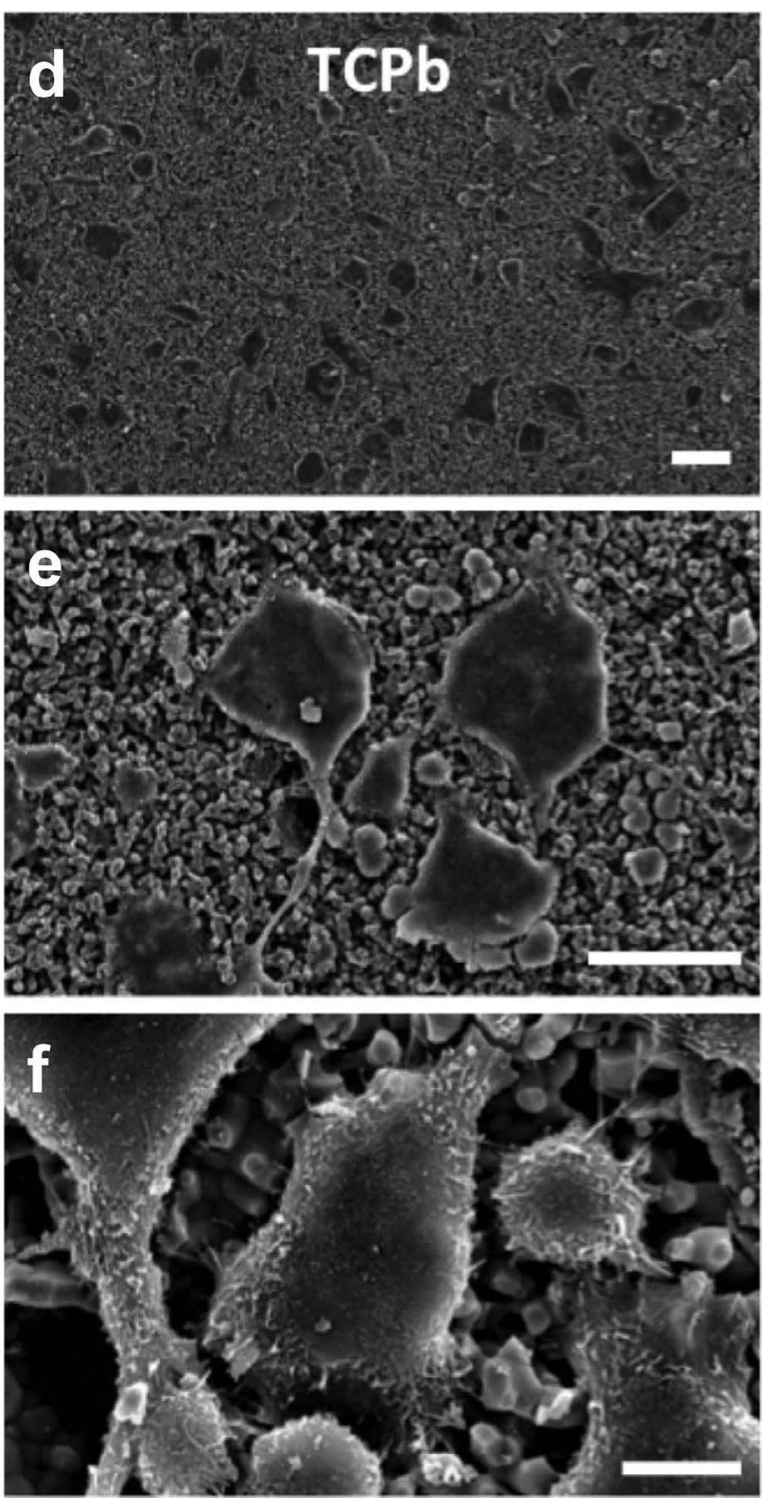

g

\section{Osteoclast Size}

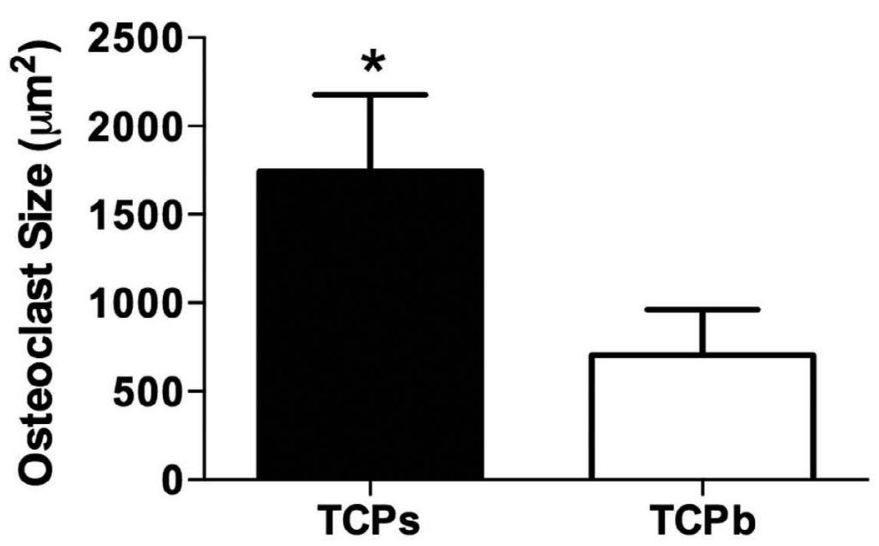

Fig. 3. SEM micrographs of osteoclast formation on (a-c) TCPs and (d-f) TCPb after $4 \mathrm{~d}$ in culture. At low magnification, osteoclasts formed on (a) TCPs are larger $(>150 \mu \mathrm{m})$ than those formed on $(\mathbf{d}) \mathrm{TCPb}(\mathrm{scale}$ bars $=100 \mu \mathrm{m}$ ). Prominent cellular filopodia connected adjacent cells and attached to the surface on (b) TCPs versus (e) TCPb (scale bars $=50 \mu \mathrm{m})$. At high magnification, (c) osteoclasts appeared tightly bound to TCPs, with spread cell membranes decorated with microvilli actively fusing with small $(<10 \mu \mathrm{m})$ mononuclear cells; $(\mathbf{f}) \mathrm{OC}$ on $\mathrm{TCPb}$ appeared rounded with less frequent fusion (scale bars $=10 \mu \mathrm{m})$. (g) Osteoclast size after 4 days culture calculated using Image J software (mean \pm s.d. of $n=3$ discs; $* p \leq 0.05$ ). 


\section{TCPs}
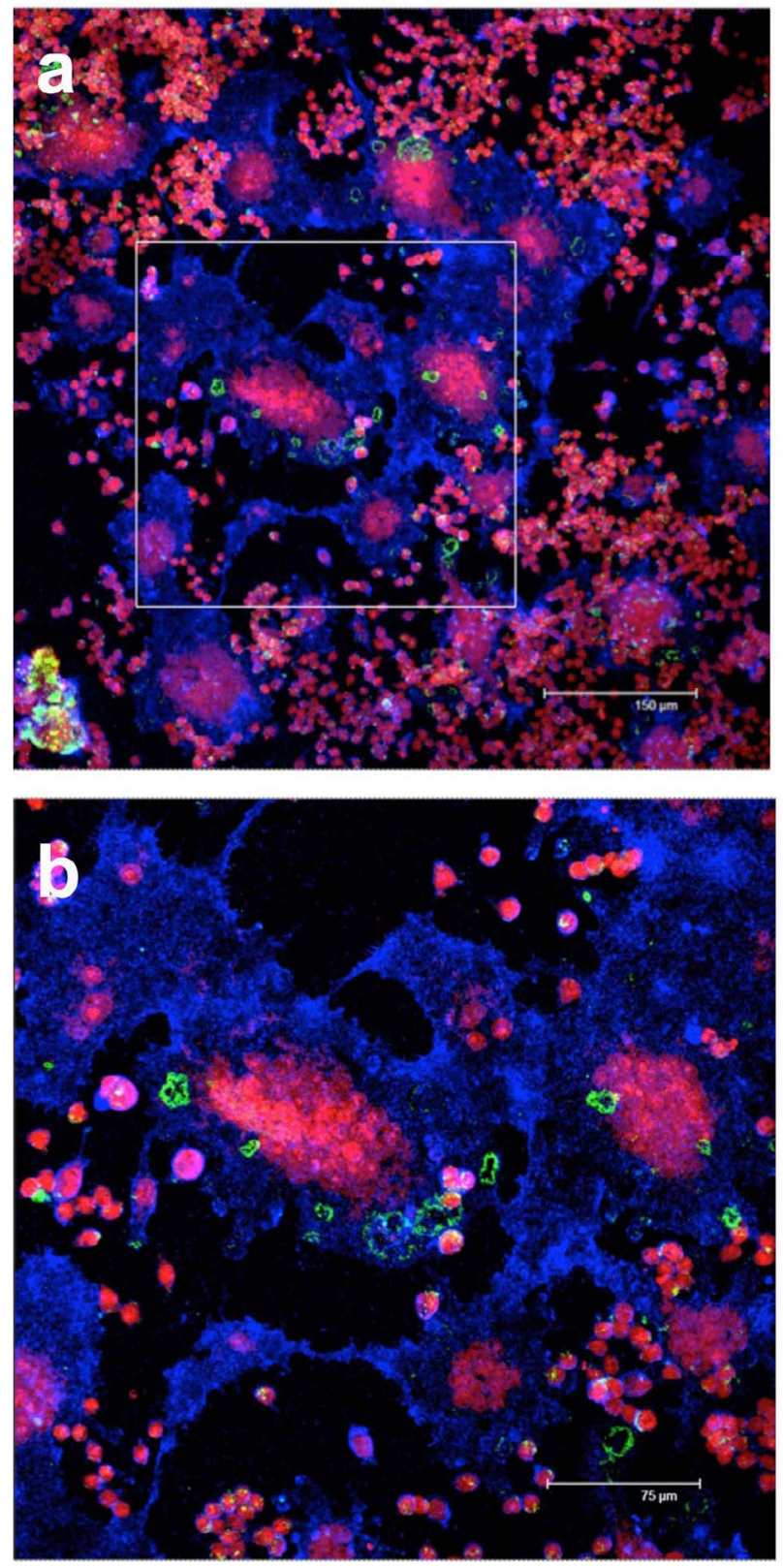

\section{$\mathrm{TCPb}$}
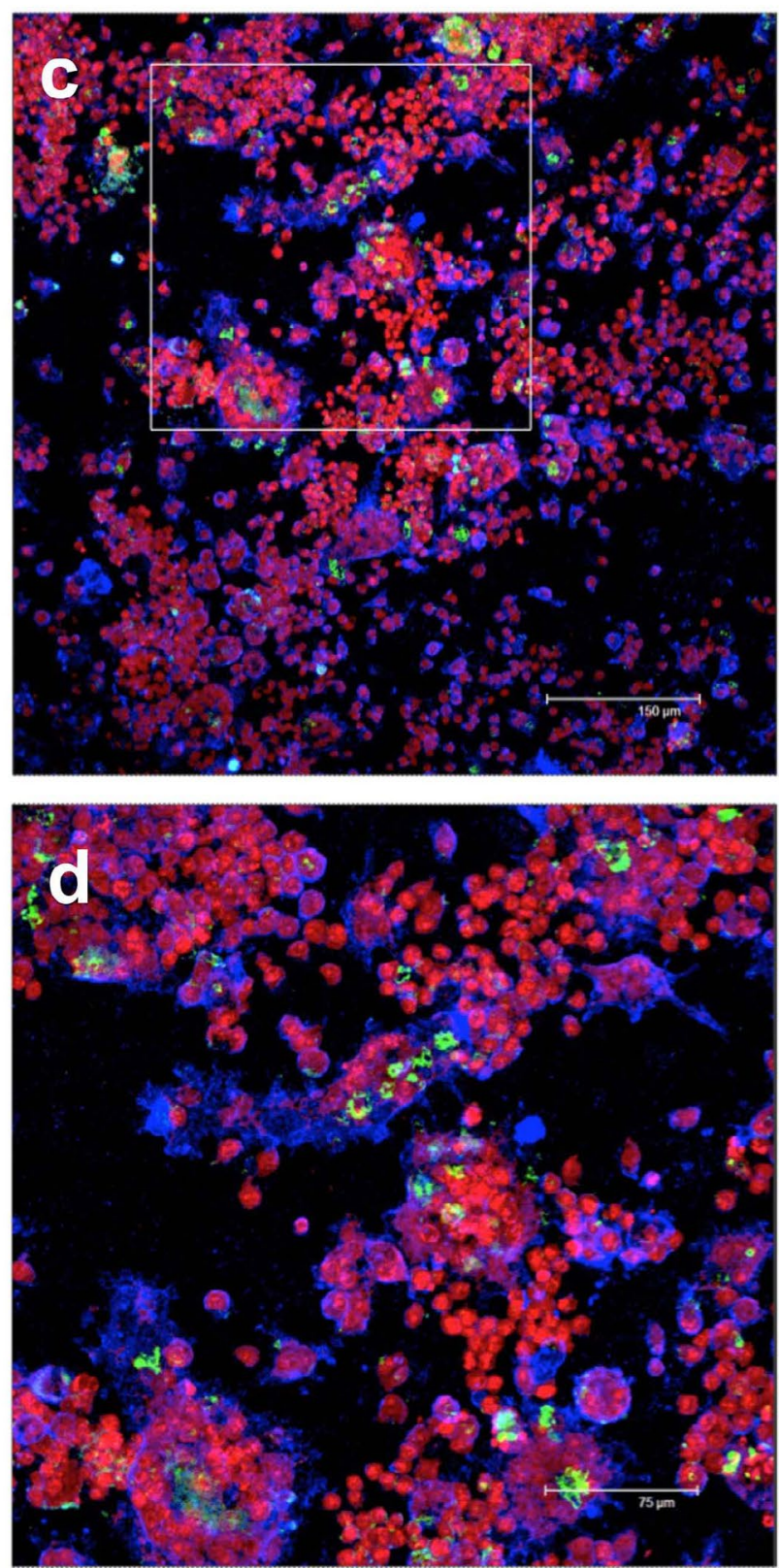

Fig. 4. Confocal fluorescent microscopy of osteoclasts formed on (a-b) TCPs and (c-d) TCPb after 4 d in culture. In low zoom images, (a) osteoclasts on TCPs were extensively interconnected by large cell membranes (CD44, blue) with highly multinucleated centres (propidium iodide, red) versus those on (c) TCPb, which were less multinucleated and less interconnected by smaller cell membranes (scale bars $=150 \mu \mathrm{m})$. At higher magnification $(\mathbf{b}, \mathbf{d})$, actin rings (phalloidin, green) were formed by cells on both materials (scale bars $=75 \mu \mathrm{m}$ ).

medium from osteoclasts formed on $\mathrm{TCPb}(p=0.002)$ or the positive hMSC control cultured in osteogenic medium $(p=0.001)$.

To compare the effects of osteoclast versus macrophage secreted osteogenic factors on osteoblast differentiation, conditioned medium from unstimulated RAW264.7 macrophages (i.e., no RANKL) was also administered to hMSC. Following culture on TCPs, osteoclastsecreted factors induced $\sim 3$ times more hMSC ALP than did macrophage-secreted factors $(p<0.001)$. Still, macrophage-secreted factors from culture on TCPs still induced significantly more hMSC ALP activity than hMSC cultured in basic medium, which was not the case for macrophage conditioned medium from culture on $\mathrm{TCPb}$.

To confirm that these effects were cell mediated and not due to the soluble chemistry of the materials or RANKL in the medium, hMSC were treated with culture medium supplemented with or without RANKL for one week, which had been similarly incubated with TCP but without RAW264.7, i.e., acellular conditioned medium. These treatments did not appreciably change hMSC ALP levels ( $p>0.99)$, confirming that the observed ALP-inductive 


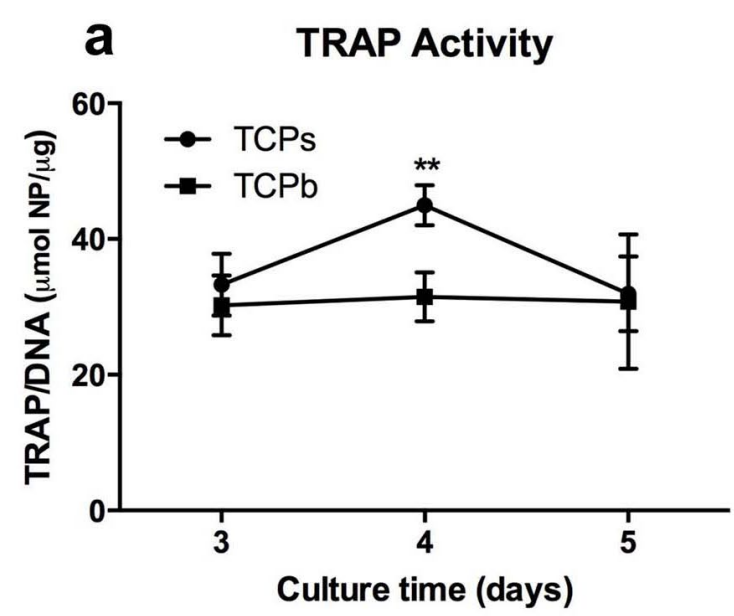

C

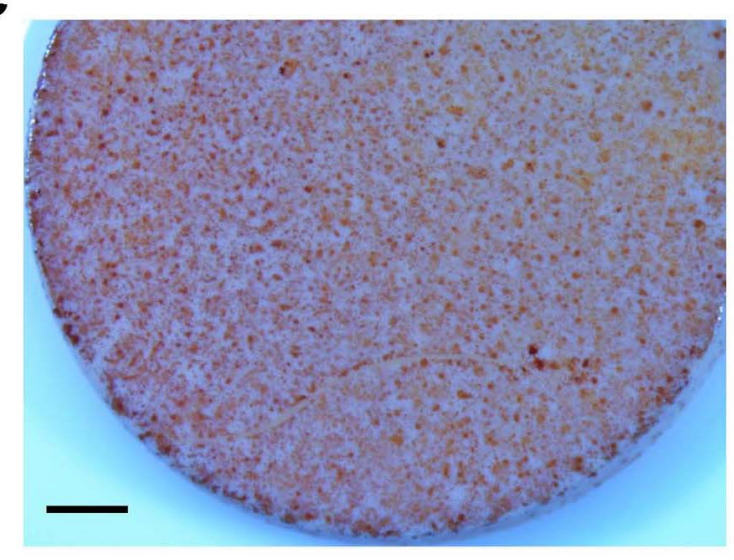

e

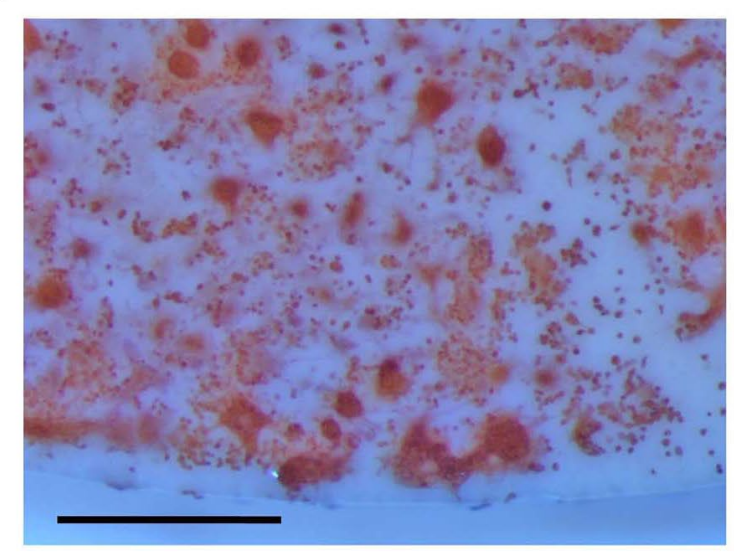

b

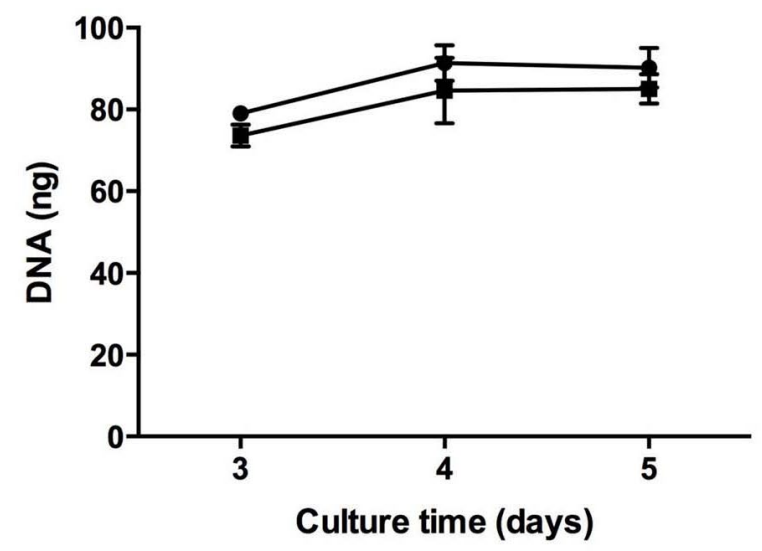

d

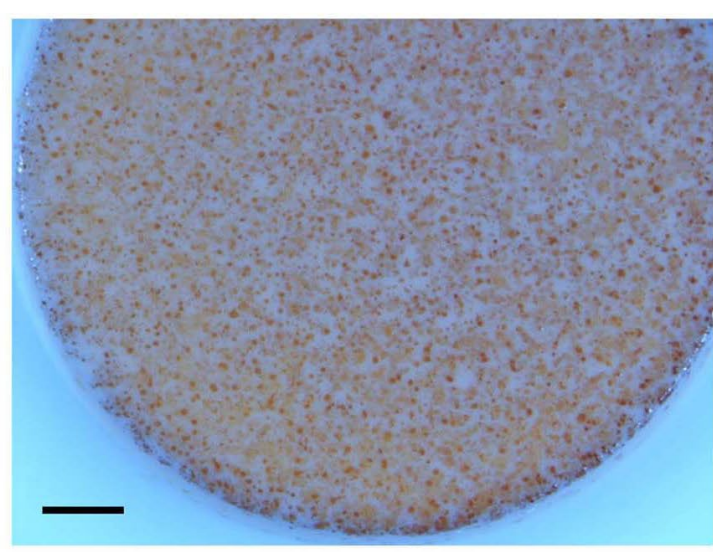

f

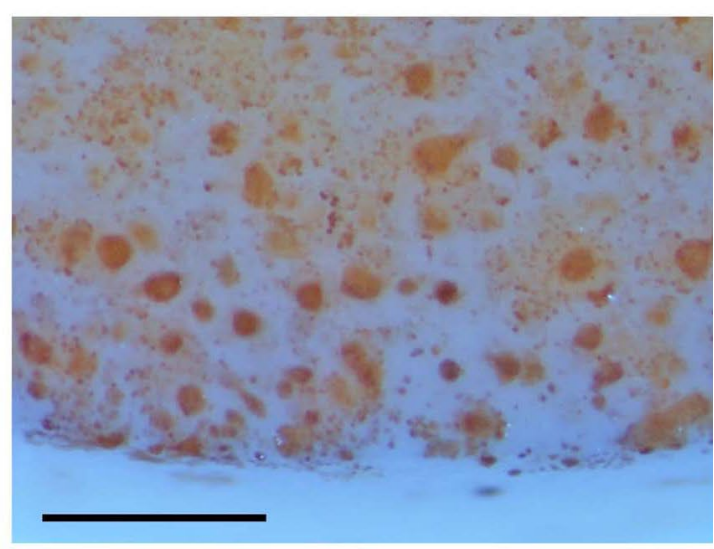

Fig. 5. Osteoclast TRAP activity on TCP discs. (a) TRAP activity per DNA in the cell lysate peaked after $4 \mathrm{~d}$ in culture on submicrostructured TCPs but remained flat on microstructured TCPb (mean \pm s.d. of $n=5$ discs. $* *$ $p \leq 0.01$ ). (b) DNA content was equivalent between materials at the same time points. Representative TRAP staining of cells cultured on $(\mathbf{c}, \mathbf{d}) \mathrm{TCPs}$ and $(\mathbf{e}, \mathbf{f}) \mathrm{TCPb}$ after $4 \mathrm{~d}$ clearly showed this difference, with most intense staining near the edge of the discs.

effects were mediated by RAW264.7 and not TCP or RANKL alone (Fig. 6).

\section{Effects of submicron-scale surface architecture on} bone formation and resorption in vivo

Histological analysis (Fig. 7a-g) and histomorphometry (Fig. 7.h,i) of intramuscular TCP implants harvested at 12 weeks demonstrated the binary effects of submicron- versus micron-scale surface architecture on bone formation: TCPs induced $20 \pm 8 \%$ area bone formation in the free implant space whereas $\mathrm{TCPb}$ formed no bone in any of the 8 test subjects (Fig. 7h). Percent area material resorption by histomorphometry was also profoundly different: resorption of TCPs was $24 \pm 5 \%$ but $\mathrm{TCPb}$ was almost entirely unresorbed $(0.3 \pm 0.1 \%)$ (Fig. 7i). Consistent throughout the TCPs samples, cuboidal osteoblasts 
Fig. 6. Biochemical ALP/DNA activity in hMSC lysate after treatment with conditioned medium (CM) for $7 \mathrm{~d}$ on tissue culture plastic. CM was collected from RAW264.7 cultured for $5 \mathrm{~d}$ on TCP discs stimulated with or without RANKL (unstimulated) to form osteoclasts or macrophages, respectively. CM collected from TCP discs without cells incubated in basic medium (BM) with or without RANKL served as acellular CM controls. hMSC cultured in $\mathrm{BM}$ or osteogenic medium (OM) without $\mathrm{CM}$ served as hMSC controls. Values represent the mean \pm s.d. of $n=4$ independent cultures of one representative hMSC donor normalised to hMSC cultured in BM. $* * p \leq 0.01, * * *$ $p \leq 0.001$.

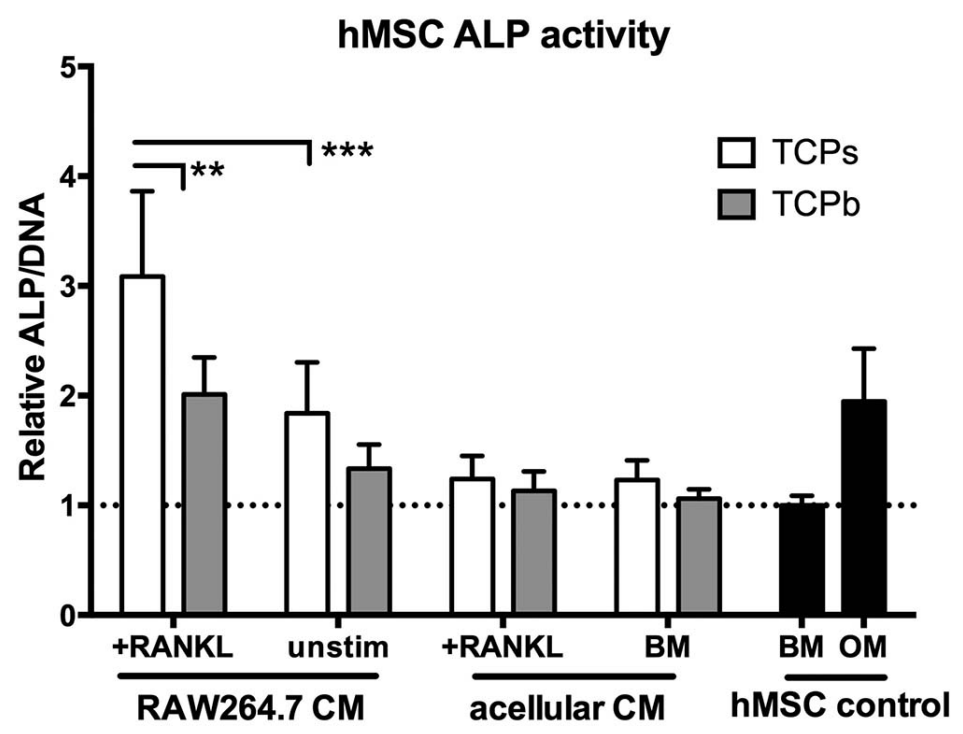

were observed forming new bone and osteocytes resided in the lacunae of mature lamellar and woven bone. Multinucleated cells morphologically resembling osteoclasts were regularly found resorbing the material, densely populating the surface that was not already occupied by new bone (Fig. $7 \mathrm{~g}$ ). These osteoclast-like cells were occasionally found on the bone but to a much lesser extent than on the material. TRAP staining to substantiate that these cells were osteoclasts was not possible due to the high curing temperature of the embedding procedure and thus inactivation of the enzyme. In striking contrast, virtually no multinucleated cells were present in any of the histological sections of $\mathrm{TCPb}$ though loose connective tissue ingrowth was apparently unhindered.

\section{Discussion}

It is the longstanding goal of biomaterial design for bone tissue repair to engineer materials that more closely simulate autologous bone. Here, we focused on replicating the aspects of bone's capacity to be remodelled and stimulate formation of new bone - i.e., material resorbability coupled with osteoinductivity. Surveying the sparse but diverse array of materials that have been shown to induce ectopic bone formation, which stands as the gold-standard demonstration of osteoinductivity, Barradas et al. (2011) suggest that surface microstructure may play a determining role in stimulating osteogenesis. But, as the authors point out, because most prior work lacks surface profile characterisation, there is no consensus on if a particular size range of surface features, such as grains and pores, can induce bone formation. Therefore, we began with the hypothesis that in order to make a material osteoinductive, the scale of surface architecture on the order of approx. $1 \mu \mathrm{m}$ may be crucial. Concerning the material resorbability, TCP provides an ideal platform, as it is commonly known to be the most resorbable of synthetic $\mathrm{CaP}$ used in the clinic.
After careful control of the synthesis technique, as we have previously described in detail (Yuan et al., 2001a; Yuan et al., 2002), TCP with either submicron- or micron-scale pores, grains and roughness was successfully synthesised while maintaining material chemistry and macroporosity. The surface profiles of the materials reflected sinusoidal undulations resulting from reciprocating pores and grains, whose amplitudes spanned a submicron or micron scale range (Fig. 1). In evaluating the intrinsic capacity of the materials to direct cell behaviour and induce bone formation in vitro and in vivo, the osteogenic effects of these surface architectural differences proved to be disparate and striking. In vitro, submicron-scale surface structure promoted osteoclast-like cell activity, fusion, and secretion of factors that amplified osteogenic differentiation of hMSC versus micron-scale topography (Figs. 2-6). In vivo, TCPs possessing submicron grains, pores, and roughness promoted extensive osteoclast-like cell colonisation and considerable ectopic bone formation (Fig. 7), comparable to the bone area of normal trabecular bone (Sato et al., 1986). In stark contrast, TCPb with micron-scale surface structure contained few osteoclastlike cells and formed no bone. Because of this, the scale of surface architecture was found to determine the osteogenic potential of TCP.

Unexpectedly, submicron surface structure determined the resorbability of the materials, also in a dichotomous manner. By histology, multinucleated osteoclast-like cells could be seen actively resorbing TCPs, whereas no such activity could be seen for TCPb. Surprisingly, TCPb with micron-scale structure was left virtually unresorbed, despite its composition of $\beta$-tricalcium phosphate (Fig. 7), shown to readily resorb in vivo (Blokhuis et al., 2000). Prior work on the mechanism of TCP resorption in vivo presents conflicting interpretations, with some groups reporting that the process is principally mediated by cellular degradation (Eggli et al., 1988), and other groups hypothesising the process is mainly due to the passive dissolution by interstitial fluids (Lu et al., 2002). 

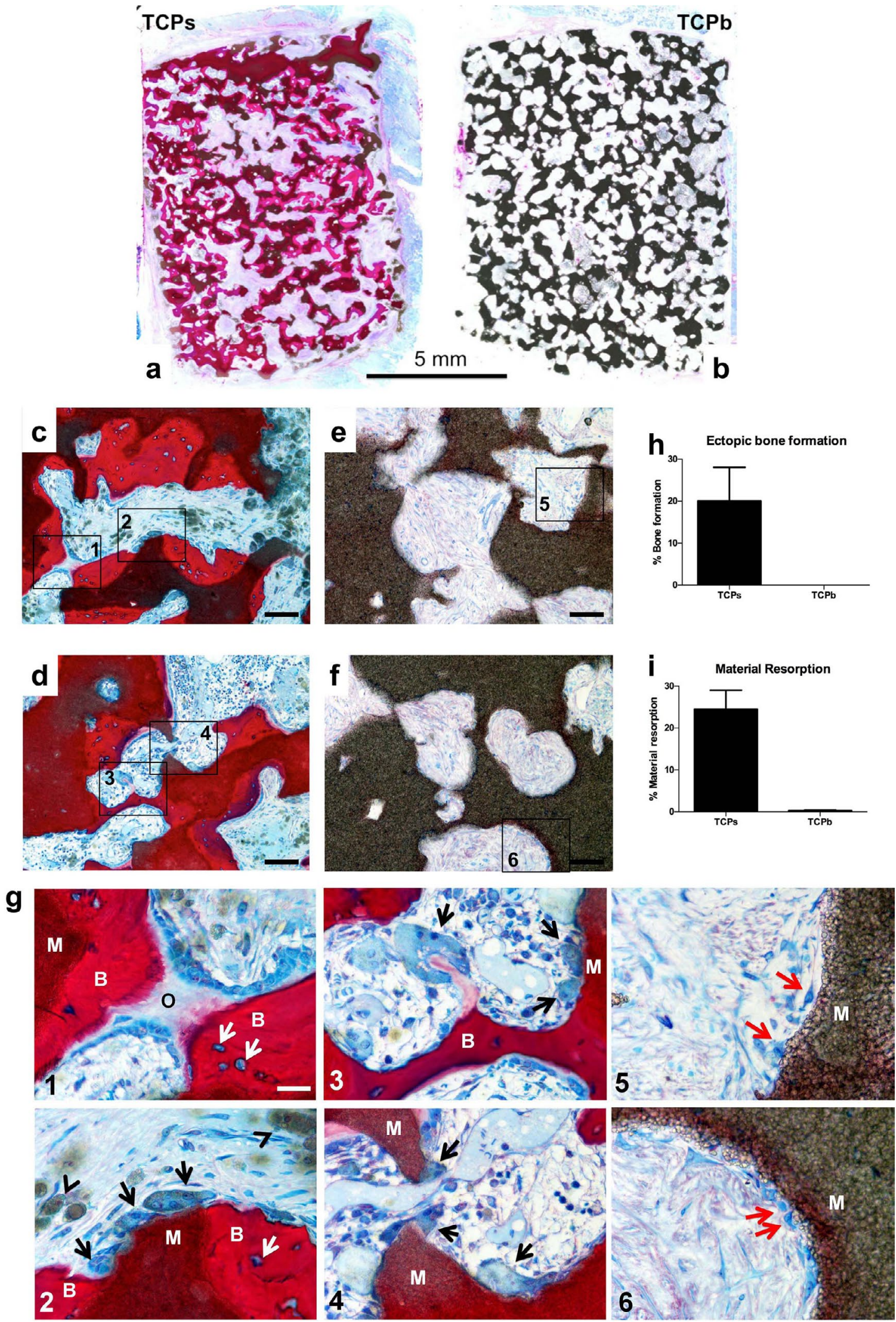

Fig. 7. Representative histological sections of macro/microporous TCP cylinders implanted in the dorsal muscle of dogs for 12 weeks. Basic fuchsin/methylene blue staining for bone (red). (a,b) Gross comparison shows ectopic bone formation by (a) TCPs and not by (b) TCPb. (c,d) Bone coverage and material fragmentation of TCPs, (e, f) No bone, no material particulate, and little tissue response in $\mathrm{TCPb}($ scale bars $=100 \mu \mathrm{m})$. $($ g) Detail insets: $1-4=\mathrm{TCPs}, 5-6=\mathrm{TCPb}$ $($ scale bar $=25 \mu \mathrm{m})$. Note: cuboidal osteoblasts form osteoid $(\mathrm{O})$ bridging mature bone $(\mathrm{B})$; osteocytes (white arrows) in characteristic lacunae $(1,2)$; multinucleated osteoclast-like cells (OCLC) (black arrows) resorbing microconcavities on TCPs material (M) between stretches of bone (2-4); phagocytosed material particulate inside OCLC (arrow heads, 2); mononuclear cells (red arrows) on $\mathrm{TCPb}$ surrounded by loose connective tissue $(5,6)$. (h) Ectopic bone formation and (i) material resorption calculated by histomorphometry (mean \pm s.d. of $n=8 \mathrm{dogs}$ ). 
In another case, TCP implanted in a non-loading cranial defect was not resorbed even after 6 months (Handschel et al., 2002). Taking into account the surface reactivity of the materials in vitro, in particular the tendency of both materials to continuously absorb rather than release $\mathrm{Ca}^{2+} / \mathrm{P}_{\mathrm{i}}$ in physiologic (supersaturated) solution even with medium refreshment, we speculate that these materials may be primarily resorbed in vivo by cellular degradation versus passive physiologic dissolution which is supported by the clear contrast of the presence of multinuclear cells and disparate resorption values. Importantly, this finding presents the possibility that surface architecture - specifically, smaller grains and pores - may promote $\mathrm{CaP}$ resorption through the differential formation of multinucleated osteoclast-like cells in addition to increasing the specific surface area available for dissolution.

In support of this inference that the scale of surface architecture may directly influence cell behaviour with direct relevance to bone formation and material resorption, we found that submicron architecture modulated osteoclast differentiation, form, and function - importantly, the secretion of osteogenic signals. These findings add to the growing understanding that surface microstructure and roughness greatly influence the formation and function of osteoclasts (Marchisio et al., 2005; Geblinger et al., 2010; Zou and Teitelbaum, 2010). Toward a potential molecular mechanism for these surface-induced effects, Makihira et al. (2007) found that submicron rough titanium upregulated osteoclast gene expression in RAW264.7 macrophages, which they attributed to elevated RANK expression, the membrane-bound receptor of RANKL. Because surface structure determines resultant material properties such as surface reactivity and topographical profile (Fig. 1), it is impossible to isolate a single factor in the cellular control by these materials. A comparison of osteoclastogenesis on unreactive materials with various surface structures might help to elucidate the effects of topography versus surface reactivity, a strategy that we are currently researching. Indeed, topographical cues as well as differences in surface reactivity of TCP tested here may have affected osteoclast behaviour synergistically. For example, extracellular $\mathrm{Ca}^{2+}$ concentration (Miyauchi et al., 1990), surface roughness (Brinkmann et al., 2012), and surface feature scale (Geblinger et al., 2010) have all been shown to greatly affect osteoclast morphology and function. In order to further characterise the osteoclast phenotype generated on the TCP materials, we performed qPCR analysis of osteoclast markers calcitonin receptor (CR), cathepsin K (CTK), vacuolar ATPase proton pump subunit V0 d2 (ATP6V0D2), osteoclast fusion protein DCSTAMP, and master osteoclast transcription factor NFATc1. All markers were upregulated in response to RANKL, thus validating the OC phenotype, but there was no clear difference between the materials (data not shown).

Despite other reports of osteoclast resorption of $\mathrm{CaP}$ and specifically TCP, no characteristic osteoclast resorption pits could be found by SEM in this study (Yamada et al., 1997; Monchau et al., 2002; Wenisch et al., 2003; Xia et al., 2006; Winkler et al., 2010). Osteoclast resorption occurs exclusively in the sealing zone bounded by cytoskeletal actin rings (Teitelbaum, 2011). Here, the size of the observed actin rings overlapped the size range of surface pores and grains, so it is possible that the micro-rough surface profile may have obscured any potential resorption. If appreciable resorption did occur, elevated $\mathrm{Ca}^{2+} / \mathrm{P}_{\mathrm{i}}$ ions in the medium might be expected, though this also was not the case (data not shown). Confoundingly, elevation of $\mathrm{Ca}^{2+} / \mathrm{P}_{\mathrm{i}}$ would likely be masked by the high affinity of TCP to rapidly absorb these ions in a supersaturated solution (Fig. 1f). Last, it is possible that appreciable resorption of sintered ceramics may require longer culture time (e.g., $28 \mathrm{~d}$ rather than $5 \mathrm{~d}$ ) (Xia et al., 2006) and larger actin rings formed by primary osteoclasts, both potential limitations of the RAW264.7 cell line in this culture model.

Still, the submicron-scale architecture of TCPs promoted osteoclast-mediated factors potent enough to induce osteoblast differentiation of hMSC without osteogenic medium supplements, confirmed in multiple donors. Given no clear evidence of osteoclast resorption and depleted $\mathrm{Ca}^{2+} / \mathrm{P}_{\mathrm{i}}$ levels in the conditioned medium, we speculate that the osteogenic effects were most likely due to osteoclast-secreted trophic factors in response to the submicrostructured surface topography. In a broader biological context, it is pertinent to note that non-resorbing osteoclasts have been identified as a source of osteogenic factors in vivo (Karsdal et al., 2007; Karsdal et al., 2008; Kreja et al., 2010), emphasising that resorption is not necessary for osteogenic signalling (Fuller et al., 2010), potentially an interesting link for non-resorbable, osteoinductive materials such as microstructured titanium. Concerning the interspecies potency of the osteogenic factors demonstrated here, Pederson et al. (2008) first reported that RAW264.7 osteoclast-like cells secrete soluble sphingosine 1 phosphate (S1P) and BMP6 which can differentiate hMSC into mineralising osteoblasts. Various other osteoclast-expressed osteogenic factors such as PDGF-BB (Kreja et al., 2010), Wnt10b (Pederson et al., 2008), other BMPs (McCullough et al., 2007), and the recently identified coupling factor CTHRC1 (Takeshita et al., 2013) may have also played a role.

Here, monocyte/macrophages cultured without RANKL on TCPs but not TCPb also induced significant hMSC ALP activity versus the negative control (Fig. 6, $p<0.05$ ), suggesting that these cells can also impact osteogenesis directly in response to osteoinductive surface structure, as others have previously suggested (Fellah et al., 2007). In vitro, inflammatory macrophages secrete osteogenic factors such as oncostatin M (OSM) (Guihard et al., 2012; Nicolaidou et al., 2012) and may also express BMPs (Champagne et al., 2002; Takebe et al., 2003; Honda et al., 2006). In vivo, Omar et al. (2011) showed that a titanium screw coated with lipopolysaccharide (LPS) to stimulate classical activation of macrophages resulted in higher bone contact when in orthotopic sites (Omar et $a l ., 2011)$ though it is unknown what impact classically activated macrophages may have on bone formation in ectopic sites.

The broad physiologic effects of surface architectural scale observed here deserve further investigation as more biomimetic materials are engineered to repair and regenerate native bone. For instance, by tuning synthesis methods to achieve pore and grain architecture on the 
nano-scale, the resulting osteogenic effects can only be speculated. At the root of this, it is unclear if one or more resulting effects of small surface architecture - such as smaller physical topography, smaller surface roughness, higher specific surface area, or surface reactivity - may play a singularly directive role in the ectopic bone formation and material resorption observed here. Additionally, surface architecture may also impact other biological processes important for bone formation such as vasculogenesis and stem cell recruitment. Interestingly, many of the same osteogenic factors that are expressed by osteoclasts and their monocyte/macrophage precursors have also been shown to chemotactically home pre-osteoblasts (OSM, PDGF, CTHRC1) and stimulate blood vessel formation (TNF- $\alpha$ ) (Kreja et al., 2010; Glass et al., 2011; Takeshita et al., 2013). For instance, invading macrophages signalled by the foreign body response may preferentially secrete cytokines important for both blood vessel formation and stem cell recruitment (e.g., pericytes) (Bergers and Song, 2005), followed by osteoblast/osteoclast differentiation (Fellah et al., 2010) - all in response to surface architecture. In this way, the biological insight derived from engineering new osteogenic material surface architectures could have important consequence for the design of other biomaterial implants where the foreign body reaction plays a key role.

\section{Conclusion}

A novel TCP ceramic composed of submicron-scale grains, pores, and surface roughness was found to potently induce ectopic bone formation, coupled with a similar rate of resorption, mimicking bone's own osteoinductivity and capacity to be remodelled. Whereas an equivalent TCP ceramic with micron-scale topographical features was unable to induce bone formation or to be resorbed, a more specific range of surface architecture for stimulating osteogenesis and material remodelling was identified. As a potential link, submicron-scale, surface structure of TCP promoted the differentiation and activity of osteoclasts resulting in the osteogenic differentiation of mesenchymal stem cells without osteogenic additives.

\section{Acknowledgements}

The authors gratefully acknowledge the support of the TeRM Smart Mix Program of the Netherlands Ministry of Economic Affairs and the Netherlands Ministry of Education, Culture and Science. This research forms part of the Project P2.04 BONE-IP of the research program of the Biomedical Materials Institute, co-funded by the Dutch Ministry of Economic Affairs. This project has also received funding from the European Union's Seventh Program for research, technological development, and demonstration under grant agreement number 241879. Special thanks are due to Davide Barbieri, Jinyi Su, Ruud Das, Rens Roosloot (Xpand Biotechnology) and Lorenzo Moroni (MIRA, University of Twente) for their technical expertise and useful discussion.

\section{References}

Akiyama N, Takemoto M, Fujibayashi S, Neo M, Hirano M, Nakamura T (2011) Difference between dogs and rats with regard to osteoclast-like cells in calciumdeficient hydroxyapatite-induced osteoinduction. J Biomed Mater Res A 96: 402-412.

Barradas AMC, Yuan H, van Blitterswijk CA, Habibovic P (2011) Osteoinductive biomaterials: current knowledge of properties, experimental models and biological mechanisms. Eur Cell Mater 21: 407-429.

Bergers G, Song S (2005) The role of pericytes in blood-vessel formation and maintenance. Neuro Oncol 7: 452-464.

Blokhuis TJ, Termaat MF, den Boer FC, Patka P, Bakker FC, Haarman HJ (2000) Properties of calcium phosphate ceramics in relation to their in vivo behavior. J Trauma 48 : 179-186.

Bohner M, Galea L, Doebelin N (2012) Calcium phosphate bone graft substitutes: Failures and hopes. J Eur Ceram Soc 32: 2663-2671.

Brinkmann J, Hefti T, Schlottig F, Spencer ND, Hall $\mathrm{H}$ (2012) Response of osteoclasts to titanium surfaces with increasing surface roughness: an et al. study. Biointerphases 7: 34 .

Champagne CM, Takebe J, Offenbacher S, Cooper LF (2002) Macrophage cell lines produce osteoinductive signals that include bone morphogenetic protein-2. Bone 30: 26-31.

Costa-Rodrigues J, Fernandes A, Lopes MA, Fernandes MH (2012) Hydroxyapatite surface roughness: complex modulation of the osteoclastogenesis of human precursor cells. Acta Biomater 8: 1137-1145.

Dalby MJ, Riehle MO, Sutherland DS, Agheli H, Curtis ASG (2004) Changes in fibroblast morphology in response to nano-columns produced by colloidal lithography. Biomaterials 25: 5415-5422.

Dalby MJ, McCloy D, Robertson M, Agheli H, Sutherland D, Affrossman S, Oreffo ROC (2006) Osteoprogenitor response to semi-ordered and random nanotopographies. Biomaterials 27: 2980-2987.

Dalby MJ, Gadegaard N, Tare R, Andar A, Riehle MO, Herzyk P, Wilkinson CDW, Oreffo ROC (2007) The control of human mesenchymal cell differentiation using nanoscale symmetry and disorder. Nat Mater 6: 997-1003.

Davison N, Yuan H, de Bruijn JD, Barrere-de Groot $\mathrm{F}$ (2012) In vivo performance of microstructured calcium phosphate formulated in novel water-free carriers. Acta Biomater 8: 2759-2769.

Dorozhkin SV (2010) Bioceramics of calcium orthophosphates. Biomaterials 31: 1465-1485.

Eggli PS, Müller W, Schenk RK (1988) Porous hydroxyapatite and tricalcium phosphate cylinders with two different pore size ranges implanted in the cancellous bone of rabbits. A comparative histomorphometric and histologic study of bony ingrowth and implant substitution. Clin Orthop Relat Res 232: 127-138.

Famery R, Richard N, Boch P (1994) Preparation of $\alpha$ - and $\beta$-tricalcium phosphate ceramics, with and without magnesium addition. Ceram Int 20: 327-336. 
Fellah BH, Josselin N, Chappard D, Weiss P, Layrolle P (2007) Inflammatory reaction in rats muscle after implantation of biphasic calcium phosphate micro particles. J Mater Sci Mater Med 18: 287-294.

Fellah BH, Delorme B, Sohier J, Magne D, Hardouin P, Layrolle P (2010) Macrophage and osteoblast responses to biphasic calcium phosphate microparticles. J Biomed Mater Res A 93: 1588-1595.

Fujibayashi S, Neo M, Kim HM, Kokubo T, Nakamura $\mathrm{T}$ (2004) Osteoinduction of porous bioactive titanium metal. Biomaterials 25: 443-450.

Fukuda A, Takemoto M, Saito T, Fujibayashi S, Neo M, Pattanayak DK, Matsushita T, Sasaki K, Nishida N, Kokubo T, Nakamura T (2011) Osteoinduction of porous Ti implants with a channel structure fabricated by selective laser melting. Acta Biomater 7: 2327-2336.

Fuller K, Ross JL, Szewczyk KA, Moss R, Chambers TJ (2010) Bone is not essential for osteoclast activation. PLoS One 5: e128.

Geblinger D, Addadi L, Geiger B (2010) Nanotopography sensing by osteoclasts. J Cell Sci 123: 18141814.

Glass GE, Chan JK, Freidin A, Feldmann M, Horwood NJ, Nanchahal J (2011) TNF-alpha promotes fracture repair by augmenting the recruitment and differentiation of muscle-derived stromal cells. Proc Natl Acad Sci USA 108: $1585-1590$.

Guihard P, Danger Y, Brounais B, David E, Brion R, Delecrin J, Richards CD, Chevalier S, Rédini F, Heymann D, Gascan H, Blanchard F (2012) Induction of osteogenesis in mesenchymal stem cells by activated monocytes/ macrophages depends on oncostatin $\mathrm{m}$ signaling. Stem Cells 30: 762-772.

Habibovic P, Yuan H, van der Valk CM, Meijer G, van Blitterswijk CA, de Groot K (2005) 3D microenvironment as essential element for osteoinduction by biomaterials. Biomaterials 26: 3565-3575.

Habibovic P, Yuan H, van den Doel M, Sees TM, van Blitterswijk CA, de Groot K (2006) Relevance of osteoinductive biomaterials in critical-sized orthotopic defect. J Orthop Res 24: 867-876.

Handschel J, Wiesmann HP, Stratmann U, Kleinheinz J, Meyer U, Joos U (2002) TCP is hardly resorbed and not osteoconductive in a non-loading calvarial model. Biomaterials 23: 1689-1695.

Honda Y, Anada T, Kamakura S, Nakamura M, Sugawara S, Suzuki O (2006) Elevated extracellular calcium stimulates secretion of bone morphogenetic protein 2 by a macrophage cell line. Biochem Biophys Res Commun 345: 1155-1160.

Jansen IDC, Vermeer JAF, Bloemen V, Stap J, Everts $\mathrm{V}$ (2012) Osteoclast fusion and fission. Calcif Tissue Int 90: $515-522$.

Karsdal MA, Martin TJ, Bollerslev J, Christiansen C, Henriksen K (2007) Are nonresorbing osteoclasts sources of bone anabolic activity? J Bone Miner Res 22: 487-494.

Karsdal MA, Neutzsky-Wulff AV, Dziegiel MH, Christiansen C, Henriksen K (2008) Osteoclasts secrete non-bone derived signals that induce bone formation. Biochem Biophys Res Commun 366: 483-488.
Kondo N, Ogose A, Tokunaga K, Umezu H, Arai K, Kudo N, Hoshino M, Inoue H, Irie H, Kuroda K, Mera H, Endo N (2006) Osteoinduction with highly purified beta-tricalcium phosphate in dog dorsal muscles and the proliferation of osteoclasts before heterotopic bone formation. Biomaterials 27: 4419-4427.

Kreja L, Brenner RE, Tautzenberger A, Liedert A, Friemert B, Ehrnthaller C, Huber-Lang M, Ignatius A (2010) Non-resorbing osteoclasts induce migration and osteogenic differentiation of mesenchymal stem cells. J Cell Biochem 109: 347-355.

LeGeros RZ (2008) Calcium phosphate-based osteoinductive materials. Chem Rev 108: 4742-4753.

Ljusberg J, Ek-Rylander B, Andersson G (1999) Tartrate-resistant purple acid phosphatase is synthesized as a latent proenzyme and activated by cysteine proteinases. Biochem J 343: 63-69.

Lu J, Descamps M, Dejou J, Koubi G, Hardouin P, Lemaitre J, Proust JP (2002) The biodegradation mechanism of calcium phosphate biomaterials in bone. $\mathrm{J}$ Biomed Mater Res 63: 408-412.

Makihira S, Mine Y, Kosaka E, Nikawa H (2007) Titanium surface roughness accelerates RANKLdependent differentiation in the osteoclast precursor cell line, RAW264. 7. Dent Mater J 26: 739-745.

Marchisio M, Di Carmine M, Pagone R, Piattelli A, Miscia S (2005) Implant surface roughness influences osteoclast proliferation and differentiation. J Biomed Mater Res B Appl Biomater 75: 251-256.

McCullough KA, Waits CA, Garimella R, Tague SE, Sipe JB, Anderson HC (2007) Immunohistochemical localization of bone morphogenetic proteins (BMPs) 2, 4, 6, and 7 during induced heterotopic bone formation. $\mathrm{J}$ Orthop Res 25: 465-472.

Miyauchi A, Hruska K A, Greenfield EM, Duncan R, Alvarez J, Barattolo R, Colucci S, Zambonin-Zallone A, Teitelbaum SL, Teti A (1990) Osteoclast cytosolic calcium, regulated by voltage-gated calcium channels and extracellular calcium, controls podosome assembly and bone resorption. J Cell Biol 111: 2543-2552.

Monchau F, Lefèvre A, Descamps M, Belquin-myrdycz A, Laffargue P, Hildebrand HF (2002) In vitro studies of human and rat osteoclast activity on hydroxyapatite, betatricalcium phosphate, calcium carbonate. Biomol Eng 19: 143-152.

Nicolaidou V, Wong MM, Redpath AN, Ersek A, Baban DF, Williams LM, Cope AP, Horwood NJ (2012) Monocytes induce STAT3 activation in human mesenchymal stem cells to promote osteoblast formation. PLoS One 7: e398: e39871.

Le Nihouannen D, Daculsi G, Saffarzadeh A, Gauthier O, Delplace S, Pilet P, Layrolle P (2005) Ectopic bone formation by microporous calcium phosphate ceramic particles in sheep muscles. Bone 36: 1086-1093.

Omar OM, Granéli C, Ekström K, Karlsson C, Johansson A, Lausmaa J, Wexell CL, Thomsen P (2011) The stimulation of an osteogenic response by classical monocyte activation. Biomaterials 32: 8190-8204.

Osborn JF, Newesely H (1980) The material science of calcium phosphate ceramics. Biomaterials 1: 108-111. 
Pederson L, Ruan M, Westendorf JJ, Khosla S, Oursler MJ (2008) Regulation of bone formation by osteoclasts involves Wnt/BMP signaling and the chemokine sphingosine-1-phosphate. Proc Natl Acad Sci USA 105: 20764-20769.

Ripamonti U, Richter PW, Nilen RWN, Renton L (2008) The induction of bone formation by smart biphasic hydroxyapatite tricalcium phosphate biomimetic matrices in the non-human primate Papio ursinus. J Cell Mol Med 12: 2609-2621.

Ripamonti U, Klar RM, Renton LF, Ferretti C (2010) Synergistic induction of bone formation by hOP-1, hTGFbeta3 and inhibition by zoledronate in macroporous coralderived hydroxyapatites. Biomaterials 31: 6400-6410.

Sato K, Wakamatsu E, Sato T, Honma T, Kotake H, Byers P (1986) Histomorphometric study of trabecular channels in normal iliac bone. Calcif Tissue Int 39: 2-7.

Sims NA, Gooi JH (2008) Bone remodeling: Multiple cellular interactions required for coupling of bone formation and resorption. Semin Cell Dev Biol 19: 444451 .

Stevens MM, George JH (2005) Exploring and engineering the cell surface interface. Science 310: 1135 1138 .

Takebe J, Champagne CM, Offenbacher S, Ishibashi K, Cooper LF (2003) Titanium surface topography alters cell shape and modulates bone morphogenetic protein 2 expression in the J774A.1 macrophage cell line. J Biomed Mater Res A 64: 207-216.

Takeshita S, Fumoto T, Matsuoka K, Park K, Aburatani H, Kato S, Ito M, Ikeda K (2013) Osteoclast-secreted CTHRC1 in the coupling of bone resorption to formation. J Clin Invest 123: 3914-3924.

Tanaka T, Saito M, Chazono M, Kumagae Y, Kikuchi T, Kitasato S, Marumo K (2010) Effects of alendronate on bone formation and osteoclastic resorption after implantation of beta-tricalcium phosphate. J Biomed Mater Res A 93: 469-474.

Teitelbaum SL (2011) The osteoclast and its unique cytoskeleton. Ann NY Acad Sci 1240: 14-17.

Theiss F, Apelt D, Brand B, Kutter A, Zlinszky K, Bohner M, Matter S, Frei C, Auer JA, von Rechenberg B (2005) Biocompatibility and resorption of a brushite calcium phosphate cement. Biomaterials 26: 4383-4394.

Wenisch S, Stahl JP, Horas U, Heiss C, Kilian O, Trinkaus K, Hild A, Schnettler R (2003) In vivo mechanisms of hydroxyapatite ceramic degradation by osteoclasts: fine structural microscopy. J Biomed Mater Res A 67: 713-718.

Winkler T, Hoenig E, Gildenhaar R, Berger G, Fritsch D, Janssen R, Morlock MM, Schilling AF (2010) Volumetric analysis of osteoclastic bioresorption of calcium phosphate ceramics with different solubilities. Acta Biomater 6: 4127-4135.

Xia Z, Grover LM, Huang Y, Adamopoulos IE, Gbureck U, Triffitt JT, Shelton RM, Barralet JE (2006) In vitro biodegradation of three brushite calcium phosphate cements by a macrophage cell-line. Biomaterials 27: 45574565 .

Yamada S, Heymann D, Bouler JM, Daculsi G (1997) Osteoclastic resorption of calcium phosphate ceramics with different hydroxyapatite/beta-tricalcium phosphate ratios. Biomaterials 18: 1037-1041.

Yuan H, Kurashina K, de Bruijn JD, Li Y, de Groot K, Zhang X (1999) A preliminary study on osteoinduction of two kinds of calcium phosphate ceramics. Biomaterials 20: $1799-1806$.

Yuan H, De Bruijn JD, Li Y, Feng J, Yang Z, De Groot $\mathrm{K}$, Zhang X (2001a) Bone formation induced by calcium phosphate ceramics in soft tissue of dogs: a comparative study between porous alpha-TCP and beta-TCP. J Mater Sci Mater Med 12: 7-13.

Yuan H, de Bruijn JD, Zhang X, van Blitterswijk CA, de Groot K (2001b) Bone induction by porous glass ceramic made from Bioglass (45S5). J Biomed Mater Res 58: 270276.

Yuan H, Van Den Doel M, Li S, Van Blitterswijk CA, De Groot K, De Bruijn JD (2002) A comparison of the osteoinductive potential of two calcium phosphate ceramics implanted intramuscularly in goats. J Mater Sci Mater Med 13: 1271-1275.

Yuan H, Fernandes H, Habibovic P, de Boer J, Barradas AMC, de Ruiter A, Walsh WR, van Blitterswijk CA, de Bruijn JD (2010) Osteoinductive ceramics as a synthetic alternative to autologous bone grafting. Proc Natl Acad Sci USA 107: 13614-13619.

Zou W, Teitelbaum SL (2010) Integrins, growth factors, and the osteoclast cytoskeleton. Ann N Y Acad Sci 1192: 27-31.

\section{Discussion with Reviewers}

M. Brennan: This paper presents a very interesting evaluation of the impact of biomaterial surface architecture on osteoclast activity in vitro and in vivo. While the increased osteogenic differentiation of MSCs by the conditioned media of osteoclasts cultured on the novel biomaterial is of immense interest, have the authors considered the direct effects of each biomaterial on MSC activity? This is important as clinical trials using cell therapies in unison with biomaterials for bone tissue engineering use MSCs.

Authors: To address the direct effect that osteoinductive surface structure might have on osteogenic differentiation, we did indeed culture hMSC from the same donors directly on the TCP materials in basic medium. However, after 7 days there was no difference in ALP activity between hMSC cultured on the materials. Barradas et al. (2013) (additional reference) recently reported upregulation of osteoblast gene markers during culture on osteoinductive TCP in osteogenic medium (+Dex); however, without a non-inductive TCP control in that study, the differential effects of osteoinductive topography could not be evaluated.

From our results versus non-inductive TCP, osteoinductive TCP surface structure directly promoted osteoclastogenesis through which osteoblast differentiation of hMSC was stimulated, suggesting that osteoclastogenesis may be critical to the process of osteoinduction. 
M. Brennan: Have the authors considered the mechanisms by which the TCPs instruct the formation of OC from monocytes/macrophages? It appears that the microporosity of the TCPs, delivers differentiation cues by mechanotransduction. Could the authors discuss how they would investigate this? Perhaps the Rho-kinase pathway is involved? Possibly the inhibition of cytoskeleton tension by using blebbistatin would be interesting?

Authors: The size of micropores has been shown elsewhere (Lee et al., 2004; additional references) to influence osteoblast differentiation, but with opposite trends: larger micropores (approx. $8 \mu \mathrm{m}$ ) tended to increase ALP activity versus smaller pores (approx. $0.2 \mu \mathrm{m}$ ) on a similar size scale as those contained in osteoinductive TCP here. As for OC differentiation, literature has mainly focused on surface roughness but this is a parameter that would change according to surface micropore and grain size. Costa-Rodrigues et al. (2012) (text reference) reported that on HA, osteoclast differentiation of human precursors was promoted by surface roughness similar to that of osteoinductive TCPs here.

To study whether osteoinductive topography stimulates OC differentiation through mechano-transduction, we have screened cytoskeletal signalling genes such as RhoA and downstream targets Src and FAK in human monocytederived OC; however, none were differentially upregulated on osteoinductive versus non-inductive TCP. Certainly inhibiting cytoskeletal reorganisation and tension at the protein level using molecules like blebbistatin or a ROCKinhibitor (McBeath et al., 2004; additional references) would be an interesting strategy to further evaluate this.

C. Evans: I imagine that these advanced materials will find their first clinical use in difficult cases, e.g., atrophic nonunions, critical size defects, etc., where soft tissue support is compromised. Have any of these types of materials been tested under compromised conditions?
Authors: Osteoinductive calcium phosphates, similar to TCPs tested here, were shown to be an effective alternative to autograft bone for the treatment of critical-sized defects, as shown by Yuan et al. (2010) and Habibovic et al. (2006) (text references). Although they have not been tested in atrophic non-unions where natural osteogenesis is impaired, osteoinductive $\mathrm{CaP}$ may hold promise in these applications similar to BMPs that are currently used in conjunction with autograft (Schmidmaier et al., 2007; additional reference) because they exert similar bone-inductive effects. Currently, these materials are used clinically for (posterolateral) spine fusion and the treatment of craniomaxillofacial defects (e.g. sinus lift and cleft palate).

\section{Additional References}

Barradas AMC, Monticone V, Hulsman M, Danoux C, Fernandes H, Tahmasebi Birgani Z, Barrère-de Groot F, Yuan H, Reinders M, Habibovic P, van Blitterswijk C, de Boer J (2013) Molecular mechanisms of biomaterial-driven osteogenic differentiation in human mesenchymal stromal cells. Integr Biol 5: 920-931.

Lee SJ, Choi JS, Park KS, Khang G, Lee YM, Lee HB (2004) Response of MG63 osteoblast-like cells onto polycarbonate membrane surfaces with different micropore sizes. Biomaterials 25: 4699-4707.

McBeath R, Pirone DM, Nelson CM, Bhadriraju K, Chen CS (2004) Cell shape, cytoskeletal tension, and RhoA regulate stem cell lineage commitment. Dev Cell 6: 483-495.

Schmidmaier G, Schwabe P, Wildemann B, Haas NP (2007) Use of bone morphogenetic proteins for treatment of non-unions and future perspectives. Injury $38 \mathrm{Suppl} 4$ : S35-41. 